TRANSACTIONS OF THE

AMERICAN MATHEMATICAL SOCIETY

Volume 356, Number 10, Pages 4019-4053

S 0002-9947(04)03461-0

Article electronically published on February 27, 2004

\title{
QUADRATIC FORMS AND PFISTER NEIGHBORS IN CHARACTERISTIC 2
}

\author{
DETLEV W. HOFFMANN AND AHMED LAGHRIBI
}

\begin{abstract}
We study Pfister neighbors and their characterization over fields of characteristic 2, where we include the case of singular forms. We give a somewhat simplified proof of a theorem of Fitzgerald which provides a criterion for when a nonsingular quadratic form $q$ is similar to a Pfister form in terms of the hyperbolicity of this form over the function field of a form $\varphi$ which is dominated by $q$. From this, we derive an analogue in characteristic 2 of a result by Knebusch saying that, in characteristic $\neq 2$, a form is a Pfister neighbor if its anisotropic part over its own function field is defined over the base field. Our result includes certain cases of singular forms, but we also give examples which show that Knebusch's result generally fails in characteristic 2 for singular forms. As an application, we characterize certain forms of height 1 in the sense of Knebusch whose quasi-linear parts are of small dimension. We also develop some of the basics of a theory of totally singular quadratic forms. This is used to give a new interpretation of the notion of the height of a standard splitting tower as introduced by the second author in an earlier paper.
\end{abstract}

\section{INTRODUCTION}

An important aspect of the algebraic theory of quadratic forms is the study of function fields of quadrics and the isotropy behaviour of quadratic forms over such function fields. Questions of this type have already, sometimes implicitly, appeared in Pfister's seminal work on quadratic forms in the 1960s, which then inspired a large number of researchers in the early 1970s (e.g., Arason, Elman, Lam, Knebusch, Wadsworth, etc.) to investigate further problems in the algebraic theory of quadratic forms where the use of function fields of quadratic forms became an indispensable tool or even a subject of study per se.

Knebusch's papers [23], 24] constitute a major advance in that they attempted for the first time to provide a self-contained in-depth study of function fields of quadratic forms and the behaviour of quadratic forms over such function fields, with particular emphasis laid upon the "generic" properties of such function fields.

Received by the editors January 21, 2003 and, in revised form, June 27, 2003.

2000 Mathematics Subject Classification. Primary 11E04; Secondary 11E81.

Key words and phrases. Quadratic form, Pfister form, Pfister neighbor, quasi-Pfister form, quasi-Pfister neighbor, function field of a quadratic form, degree of a quadratic form, splitting tower, height of a quadratic form, singular quadratic form.

Both authors have been supported in part by the European research networks FMRX-CT970107 and HPRN-CT-2002-00287 "Algebraic K-Theory, Linear Algebraic Groups and Related Structures", and by the program INTAS 99-00817 "Linear Algebraic Groups and Related Linear and Homological Structures". 
These two papers led to a wealth of new results, and opened many possible pathways for further research by asking important questions, some of which have only just recently been answered using new techniques developed partly in the wake of Voevodsky's work on the Milnor conjecture. Knebusch's papers were followed in the early 1980s by various articles on function fields most notably by Fitzgerald, Elman-Lam, Elman-Lam-Wadsworth, etc.

Starting in the 1990s, function field questions once again became of interest due to Merkurjev's construction of fields with $u$-invariant equal to any given even number (the $u$-invariant of a field $F$ being the supremum of the dimensions of anisotropic forms over $F$ which are torsion in the Witt ring of $F$ ). Of particular importance became the question of when an anisotropic form over $F$ becomes isotropic over the function field $F(\psi)$ of another form $\psi$. This question has been studied extensively by the present authors, Izhboldin, Karpenko, Merkurjev, Vishik, etc.

Most of the above-mentioned studies have one thing in common: they all deal initially only with quadratic forms over fields of characteristic $\neq 2$. Several of the results obtained have been extended to quadratic forms over fields of characteristic 2 (see, e.g., [1], [6], [7], [8], 10], 11], 12], [26], 32]). However, in most of these articles the emphasis has been on nonsingular quadratic forms (forms without radical) or regular forms (nonsingular forms or those with nonvanishing radical of dimension $\leq 1)$.

Only recently have there been efforts to develop a theory of function fields of quadratic forms in characteristic 2 which systematically includes the case of singular forms, following the initial ideas developed in [23], 24]. In [25], Knebusch embarks on a study of the generic aspects of such a theory (places, specializations, etc.) which in its scope goes far beyond previous attempts. The aspect of the isotropy of quadratic forms over function fields of quadratic forms (singular or not) and the behaviour of forms over their own function field have been treated systematically in [28], [29], [31]. It is this latter aspect which will constitute the centerpiece of the present article.

The central topic of this paper is the extension to characteristic 2 of two important theorems concerning Pfister forms and Pfister neighbors, and whose characteristic $\neq 2$ versions are due to Fitzgerald [15] and Knebusch [24]. In characteristic $\neq 2$, Pfister forms are tensor products of a finite number of binary (regular) forms and they play a central role in the algebraic theory of quadratic forms. A Pfister neighbor of a Pfister form is a form similar to an orthogonal summand of this Pfister form and of dimension greater than half the dimension of this Pfister form. It turns out that much of the information about a Pfister form can already be retrieved from information about a neighbor of this Pfister form and vice versa. Now anisotropic Pfister forms and neighbors can intrinsically be characterized by how they behave over their own function field. In fact, an anisotropic form is similar to a Pfister form iff the form is hyperbolic over its own function field ([23], 38]), and an anisotropic form $\varphi$ over $F$ is a Pfister neighbor if its anisotropic part over its own function field is defined over the ground field $F$; i.e., there exists a form $\psi$ over $F$ such that $\left(\varphi_{F(\varphi)}\right)_{\text {an }} \cong \psi_{F(\varphi)}$. This latter statement is due to Knebusch [24] Th. 7.13] and also follows from a more general result due to Fitzgerald [15, Th. 1.6] which essentially can be stated as saying that if an anisotropic form $\varphi$ over $F$ becomes hyperbolic over $F(q)$ for some form $q$ over $F$, and if $q$ is in a certain sense "big enough" compared with $\varphi$, then $\varphi$ is similar to a Pfister form. 
The plan of the paper is as follows. The next three sections provide an account of some basic facts on quadratic forms in characteristic 2 and list some of the deeper results which we will use in our proofs. Since general references to facts about singular forms are relatively sparse in the literature, we decided to include, sometimes in quite some detail, rather basic and elementary results which might hitherto have gone unnoticed or which might belong to mathematical folklore and for which we did not find a suitable reference.

Section 2 deals with Witt cancellation and Witt decomposition which yield a notion of Witt equivalence based on comparing anisotropic parts of (possibly singular) forms.

Section 3 deals with the notions of subform (i.e., an orthogonal summand of another form) and of dominated form (i.e., the restriction of a form to a subvector space of the underlying vector space of another form), as well as the notion of a nonsingular completion of a singular form (i.e., a nonsingular form of minimal dimension dominating a given singular form). These notions of domination and nonsingular completion plus our notion of Witt equivalence provide very effective tools (in particular, the "completion lemma" 3.9) in order to circumnavigate the use of Witt cancellation in the original proofs of Knebusch's and Fitzgerald's theorems in characteristic $\neq 2$. The main result of Section 3 is Theorem 3.11 which is the most general version in characteristic 2 of determining the maximal dimension of a common dominated form of two forms in terms of the dimensions of totally isotropic subspaces of these two forms and of their orthogonal difference.

In Section 4, we give definitions of various notions and objects which will appear in the statements and proofs of our results (Pfister form, function field, splitting tower, degree, Arf-invariant and Clifford algebra, etc.), and we will state several deep results used in the proofs, some of them rather recent (e.g., [6], [7], [18]).

Section 5 is almost entirely devoted to the proof of Fitzgerald's theorem in characteristic 2. Our version of the theorem was formulated so that it also includes the case of singular forms (dominated by nonsingular forms) and their function fields.

Fitzgerald's theorem is then applied in Section 6 to prove a characteristic 2 version of Knebusch's theorem. It should be remarked that Knebusch's theorem carries over to characteristic 2 for nonsingular forms without much difficulty, but we construct various types of counterexamples showing that it fails in general for singular forms. However, we conjecture that if the dimension $s$ of the radical of an anisotropic form $q$ over $F$ is less than $\operatorname{dim} q / 2$, then Knebusch's theorem holds, and we prove this conjecture for $s \leq 4$ and for a particular case when $s=5$ (Theorem 6.6).

As an application, we classify in Section 7 forms of nondefective height 1 (i.e., forms which are not totally singular but whose anisotropic parts over their own function fields are totally singular) provided they satisfy the same hypotheses on the radical as the forms in our version of Knebusch's theorem (Theorem 7.5).

Finally, Section 8 stands somewhat apart as it deals almost exclusively with totally singular forms. This section can be read independently of the others (perhaps with the exception of Theorem 8.16), but two or three of its results will be used in earlier sections (and might be taken for granted at that moment). It turns out that one can develop a meaningful theory of totally singular quadratic forms in characteristic 2 by almost altogether ignoring the theory of nonsingular resp. nontotally singular forms. In particular, one has notions of forms which we call 
quasi-Pfister forms and quasi-Pfister neighbors with properties which resemble in a surprising way many of the better known properties of "classical" Pfister forms and Pfister neighbors. Since Pfister forms and neighbors are part of the main theme of the present paper, it is for this reason too that we decided to include this section on totally singular forms. It should be noted that some of the results in this last section or variations of these results have first been shown by the second author in [30. where also other aspects of totally singular forms have been investigated. Our approach here, however, is different and based on the notion of what we call the norm field of a totally singular form. This norm field is a totally inseparable multiquadratic extension of the field of squares of the base field which is in a natural way associated to a totally singular form and which encodes various properties of that form. As an application of these techniques, we give in Theorem 8.16 (resp. Theorem 4.5) an interpretation of the standard height (as defined in [29]) of an anisotropic form (not necessarily totally singular) as the maximal height of a field tower such that the dimensions of the anisotropic parts of this form over the fields along this tower are strictly decreasing.

For all basic results on forms in characteristic 2 which we mention without reference, we refer the reader to [10], [11], 12], and sections 1.4 and 2.4 in [35].

\section{Witt CAnCEllation AND Witt Decomposition}

Let $F$ be a field of characteristic 2. A quadratic form over $F$ is a pair $(V, q)$ of an $F$-vector space $V$ and a function $q: V \rightarrow F$ such that

(1) $q(a x)=a^{2} q(x)$ for all $a \in F$ and all $x \in V$;

(2) there exists a symmetric bilinear form $b_{q}: V \times V \rightarrow F$ such that $q(x+y)-q(x)-q(y)=b_{q}(x, y)$ for all $x, y \in V$.

It is well known that there exists a basis $e_{i}, f_{i}, g_{j}$ and elements $a_{i}, b_{i}, c_{j} \in F$, $1 \leq i \leq r, 1 \leq j \leq s$, of $V$ with $\operatorname{dim} V=2 r+s$ such that

$$
q(v)=\sum_{i=1}^{r}\left(a_{i} x_{i}^{2}+x_{i} y_{i}+b_{i} y_{i}^{2}\right)+\sum_{j=1}^{s} c_{j} z_{j}^{2}
$$

for all $v=\sum_{i=1}^{r}\left(x_{i} e_{i}+y_{i} f_{i}\right)+\sum_{j=1}^{s} z_{j} g_{j}$. For a quadratic form given by such a polynomial we will write

$$
q=\left[a_{1}, b_{1}\right] \perp\left[a_{2}, b_{2}\right] \perp \cdots \perp\left[a_{r}, b_{r}\right] \perp\left\langle c_{1}, \cdots, c_{s}\right\rangle
$$

for short. If $q$ is written in this way, we will call it a normalized form. We will often and implicitly use certain isometries which are rather obvious or easy to prove. We will refer to them as standard relations and they are the following:

$$
\begin{gathered}
\langle a\rangle \cong\left\langle x^{2} a\right\rangle,[a, b] \cong\left[a x^{2}, b x^{-2}\right] \text { for all } a, b \in F, x \in F^{*} ; \\
\langle a, b\rangle \cong\langle a, a+b\rangle \cong\langle b, a\rangle,[a, b] \cong[a, a+b+1] \cong[b, a] \text { for all } a, b \in F \\
{[a, b] \perp\langle c\rangle \cong[a+c, b] \perp\langle c\rangle \text { for all } a, b, c \in F} \\
{[a, b] \perp[c, d] \cong[a+c, b] \perp[c, b+d] \text { for all } a, b, c, d \in F .}
\end{gathered}
$$

Remark 2.1. In many books on forms in characteristic 2 , the notation $\left\langle c_{1}, \cdots, c_{s}\right\rangle$ is used for the bilinear form given by $b(X, Y)=\sum_{i=1}^{s} c_{i} x_{i} y_{i}$ for $X=\left(x_{1}, \cdots, x_{s}\right)$, $Y=\left(y_{1}, \cdots, y_{s}\right)$. Since we consider almost exclusively quadratic forms and in order to simplify notation, we use the notation $\left\langle c_{1}, \cdots, c_{s}\right\rangle$ for the diagonal quadratic form $q(X)=b(X, X)$, and we denote the bilinear form by $\left\langle c_{1}, \cdots, c_{s}\right\rangle_{b}$ instead. 
Let $K / F$ be a field extension. If $\varphi$ is a form over $F$, then we will denote by $\varphi_{K}$ the form obtained after scalar extension from $F$ to $K$. We write $D_{K}(\varphi)$ for the elements represented nontrivially by $\varphi$ over $K, D_{K}^{*}(\varphi)=D_{K}(\varphi) \cap K^{*}$, and $G_{K}(\varphi)$ for the group of similarity factors of $\varphi$ over $K$, i.e., $G_{K}(\varphi)=\left\{a \in K^{*} \mid a \varphi_{K} \cong \varphi_{K}\right\}$.

The subspace of $V$ spanned by the $g_{i}$ as above is nothing but the radical $\operatorname{rad}(q)=$ $V^{\perp}$ of $q$, i.e., $\operatorname{span}_{V / F}\left\{g_{1}, \cdots, g_{s}\right\}=\left\{x \in V \mid b_{q}(x, y)=0 \forall y \in V\right\}$. If $s>0$, then $q_{r}=\left[a_{1}, b_{1}\right] \perp\left[a_{2}, b_{2}\right] \perp \cdots \perp\left[a_{r}, b_{r}\right]$ is generally not determined uniquely up to isometry. However, $q_{s}=\left\langle c_{1}, \cdots, c_{s}\right\rangle$ is always determined uniquely up to isometry as the radical $V^{\perp}$ is determined uniquely, and $q_{s}$ in the above representation will be called the quasi-linear part of $q$ and denoted by $\mathrm{ql}(q)$. In particular, $r$ and $s$ are determined uniquely and we say that $q$ is of type $(r, s)$. We note that the type of $q$ does not change after passing to an extension field.

$q$ is said to be nonsingular if $s=0$. If $s \neq 0$ (and $r=0$ ), then $q$ is said to be singular (totally singular). $q$ will be called nondefective if $q$ is nonsingular or if $\mathrm{ql}(q)$ is anisotropic.

Consider another totally singular form $q_{s}^{\prime}=\left\langle c_{1}^{\prime}, \cdots, c_{s}^{\prime}\right\rangle$. Then it is an easy exercise to show that $q_{s} \cong q_{s}^{\prime}$ (i.e., the two quadratic forms are isometric) if and only if $\operatorname{span}_{F / F^{2}}\left\{c_{1}, \cdots, c_{s}\right\}=\operatorname{span}_{F / F^{2}}\left\{c_{1}^{\prime}, \cdots, c_{s}^{\prime}\right\}$, i.e., the elements $c_{i}$ generate the same subvector space of the vector space $F$ over the field $F^{2}$ as the elements $c_{i}^{\prime}$. In particular, if $\left\{d_{1}, \cdots, d_{t}\right\}, t \leq s$, is a basis of $\operatorname{span}_{F / F^{2}}\left\{c_{1}, \cdots, c_{s}\right\}$, then $q_{s} \cong\left\langle d_{1}, \cdots, d_{t}, 0, \cdots, 0\right\rangle$, with $\left\langle d_{1}, \cdots, d_{t}\right\rangle$ anisotropic. We call $\left\langle d_{1}, \cdots, d_{t}\right\rangle$ the anisotropic part of $q_{s}$. This anisotropic part is, by the above, uniquely determined up to isometry, and in fact we can choose the elements $d_{i}$ among the $c_{i}$. We thus get:

Lemma 2.2. Let $q$ be a quadratic form over $F$ of type $(r, s)$. Let $E$ be any field extension of $F$. Then the anisotropic part of $\mathrm{ql}\left(q_{E}\right)$ is defined over $F$.

Proof. Let $\mathrm{ql}(q)=\left\langle c_{1}, \cdots, c_{s}\right\rangle, c_{i} \in F$. After reindexation, let $\left\{c_{1}, \cdots, c_{t}\right\}, t \leq s$, be a basis of $\operatorname{span}_{E / E^{2}}\left\{c_{1}, \cdots, c_{s}\right\}$. Then the anisotropic part of $\mathrm{ql}\left(q_{E}\right)$ is isometric to $\left\langle c_{1}, \cdots, c_{t}\right\rangle_{E}$ and thus defined over $F$.

Remark 2.3. The above observation, as elementary as it is, seems to have gone to some extent unnoticed. This is evidenced, for example, in 8. 1.3], where a proof is found for a rather special case of the above lemma, namely for $E$ an inseparable quadratic extension and $q$ totally singular.

A hyperbolic plane $\mathbb{H}$ is a nonsingular binary form isometric to $[0,0]$, and a nonsingular form is said to be hyperbolic if it is isometric to an orthogonal sum of hyperbolic planes. One verifies readily that if $q=q_{r} \perp q_{s}$ is a quadratic form with $q_{r}$ nonsingular and $q_{s}$ totally singular and anisotropic, and if $q$ is isotropic, then $q \cong[0,0] \perp q_{r}^{\prime} \perp q_{s}$ with $q_{r}^{\prime}$ nonsingular.

We thus get the following result which is an analogue in characteristic 2 to the usual Witt decomposition in characteristic $\neq 2$.

Proposition 2.4. Let $q$ be a quadratic form over $F$. Then $q \cong i \times \mathbb{H} \perp \tilde{q}_{r} \perp \tilde{q}_{s} \perp$ $j \times\langle 0\rangle$ with $\tilde{q}_{r}$ nonsingular, $\tilde{q}_{s}$ totally singular and $\tilde{q}_{r} \perp \tilde{q}_{s}$ anisotropic. The form $\tilde{q}_{r} \perp \tilde{q}_{s}$ is uniquely determined up to isometry. In particular, $i$ and $j$ are uniquely determined.

In view of this proposition, we call $\tilde{q}_{r} \perp \tilde{q}_{s}$ the anisotropic part of $q$ and we write $q_{\text {an }}$ for short. $i$ resp. $j$ will be called the Witt index resp. defect of $q$, denoted by 
$i_{W}(q)=i$ and $i_{\mathrm{ql}}(q)=j$. Furthermore, $i \times \mathbb{H} \perp q_{\text {an }}$ will be called the nondefective part of $q$. For nondefective forms (the case $j=0$ ), this result can be found in [9] page 160] or in [22, page 283]. Note that $i_{W}(q)+i_{\mathrm{ql}}(q)$ is exactly the dimension of any maximal totally isotropic subspace of $q$ (a subspace $W$ of the underlying vector space $V$ of $q$ is called totally isotropic if $q(w)=0$ for all $w \in W)$. Therefore, we call $i_{\mathrm{ti}}(q)=i_{W}(q)+i_{\mathrm{ql}}(q)$ the total isotropy index of $q$.

We call two forms $\varphi$ and $\psi$ Witt-equivalent, denoted by $\varphi \sim \psi$, if $\varphi_{a n} \cong \psi_{a n}$. If $\varphi$ and $\psi$ are nonsingular, then $\varphi \sim \psi$ iff $\varphi \perp-\psi$ is hyperbolic, the usual definition of Witt equivalence. Thus, the notion of Witt equivalence for nonsingular forms extends in a natural way to forms which are not necessarily nonsingular.

To prove the proposition, we need the following result on Witt cancellation in characteristic 2 (cf. [22 Prop. 1.2]).

Proposition 2.5. Let $q$ and $q^{\prime}$ be quadratic forms of the same dimension and let $\varphi$ be a nonsingular quadratic form. If $q \perp \varphi \cong q^{\prime} \perp \varphi$, then $q \cong q^{\prime}$.

We also need the following cancellation result.

Lemma 2.6. Let $q$ and $q^{\prime}$ be nondefective quadratic forms of the same dimension. If $q \perp j \times\langle 0\rangle \cong q^{\prime} \perp j \times\langle 0\rangle$, then $q \cong q^{\prime}$.

Proof. Write $q \cong m \times \mathbb{H} \perp q_{0}$ and $q^{\prime} \cong m^{\prime} \times \mathbb{H} \perp q_{0}^{\prime}$ with $q_{0}$ and $q_{0}^{\prime}$ anisotropic and, say, $m^{\prime} \geq m$. Put $n=m^{\prime}-m$ and $q_{1}^{\prime}=n \times \mathbb{H} \perp q_{0}^{\prime}$. By Witt cancellation for common nonsingular orthogonal summands (Proposition 2.5), it suffices to show that for $\varphi=q_{0} \perp j \times\langle 0\rangle$ and $\varphi^{\prime}=q_{1}^{\prime} \perp j \times\langle 0\rangle$, we have that $\varphi \cong \varphi^{\prime}$ implies $q_{0} \cong q_{1}^{\prime}$.

Let $V=W \oplus U$ and $V^{\prime}=W^{\prime} \oplus U^{\prime}$ be the underlying vector spaces of $\varphi$ and $\varphi^{\prime}$, respectively, such that $\left.\varphi\right|_{W}=q_{0},\left.\varphi^{\prime}\right|_{W^{\prime}}=q_{1}^{\prime}$, and $\left.\varphi\right|_{U}=j \times\langle 0\rangle=\left.\varphi^{\prime}\right|_{U^{\prime}}$.

Now let $\sigma: V \rightarrow V^{\prime}$ be a vector space isomorphism which is an isometry of $\varphi$ and $\varphi^{\prime}$, i.e., $\varphi^{\prime}(\sigma(x))=\varphi(x)$ for all $x \in V$. Let $\pi: V^{\prime}=W^{\prime} \oplus U^{\prime} \rightarrow W^{\prime}$ be the projection onto $W^{\prime}$, and define $\tau: W \rightarrow W^{\prime}$ by $\tau=\left.\pi \circ \sigma\right|_{W}$.

If $w \in W$ and $\sigma(w)=w^{\prime}+u^{\prime}, w^{\prime} \in W^{\prime}, u^{\prime} \in U^{\prime}$, then $\tau(w)=w^{\prime}$ and thus

$$
q_{1}^{\prime}(\tau(w))=\varphi^{\prime}(\tau(w))=\varphi^{\prime}\left(w^{\prime}\right)=\varphi^{\prime}\left(w^{\prime}+u^{\prime}\right)=\varphi^{\prime}(\sigma(w))=\varphi(w)=q_{0}(w),
$$

where the third equality follows from the fact that $u^{\prime} \in U^{\prime} \subset V^{\prime \perp}$ and $\varphi^{\prime}\left(u^{\prime}\right)=0$. To show that $\tau$ is an isometry, it suffices to show that $\tau$ is bijective. Now if $0 \neq w \in W$, then $q_{0}(w) \neq 0$ as $q_{0}$ is anisotropic. But then $0 \neq q_{1}^{\prime}(\tau(w))=q_{0}(w)$, hence $\tau(w) \neq 0$ and $\tau$ is therefore injective. The bijectivity follows as $\operatorname{dim} W=\operatorname{dim} W^{\prime}$.

Proof of Proposition 2.4. We only have to prove uniqueness of the decomposition. Now $\tilde{q}_{s} \perp j \times\langle 0\rangle \cong \mathrm{ql}(q)$, and it follows immediately that $j$ is uniquely determined and so is $\tilde{q}_{s}$ (up to isometry). So it suffices to show that if $i \times \mathbb{H} \perp \tilde{q}_{r} \perp \tilde{q}_{s} \perp$ $j \times\langle 0\rangle \cong i^{\prime} \times \mathbb{H} \perp \tilde{q}_{r}^{\prime} \perp \tilde{q}_{s} \perp j \times\langle 0\rangle$ with $\tilde{q}_{r}, \tilde{q}_{r}^{\prime}$ nonsingular and $\tilde{q}_{r} \perp \tilde{q}_{s}, \tilde{q}_{r}^{\prime} \perp \tilde{q}_{s}$ anisotropic, then $\tilde{q}_{r} \perp \tilde{q}_{s} \cong \tilde{q}_{r}^{\prime} \perp \tilde{q}_{s}$.

By Lemma 2.6, we have $i \times \mathbb{H} \perp \tilde{q}_{r} \perp \tilde{q}_{s} \cong i^{\prime} \times \mathbb{H} \perp \tilde{q}_{r}^{\prime} \perp \tilde{q}_{s}$. But then it follows immediately from Proposition 2.5 that $i=i^{\prime}$ and $\tilde{q}_{r} \perp \tilde{q}_{s} \cong \tilde{q}_{r}^{\prime} \perp \tilde{q}_{s}$.

\section{SUbFORMS AND DOMINATED FORMS}

Lemma 3.1. (i) Let $(q, V)$ and $(\varphi, W)$ be quadratic spaces over $F$. Then the following are equivalent. 
(1) There exists an injective isometry $t:(q, V) \rightarrow(\varphi, W)$ (i.e., $t$ is an injective $F$-linear map $V \rightarrow W$ with $\varphi(t(x))=q(x)$ for all $x \in V)$.

(2) There exist nonsingular forms $q_{r}$ and $\tau$, nonnegative integers $s^{\prime} \leq s \leq s^{\prime \prime}$, $c_{i} \in F\left(1 \leq i \leq s^{\prime \prime}\right)$ and $d_{j} \in F\left(1 \leq j \leq s^{\prime}\right)$ such that $q \cong q_{r} \perp\left\langle c_{1}, \cdots, c_{s}\right\rangle$ and

$$
\varphi \cong q_{r} \perp \tau \perp\left[c_{1}, d_{1}\right] \perp \cdots \perp\left[c_{s^{\prime}}, d_{s^{\prime}}\right] \perp\left\langle c_{s^{\prime}+1}, \cdots, c_{s^{\prime \prime}}\right\rangle .
$$

(ii) Suppose that the equivalent conditions in (i) hold. With the same notation, let $U=t(V)^{\perp}=\left\{x \in W \mid b_{\varphi}(x, t(V))=0\right\}$. Then $\left.\varphi\right|_{U} \cong \tau \perp\left\langle c_{1}, \cdots, c_{s^{\prime \prime}}\right\rangle$.

Proof. Part (ii) follows readily from part (i) and is left to the reader. As for part (i), the implication $(2) \Rightarrow(1)$ is clear.

To prove the converse, we may assume that $V \subset W$ and that $t$ is the canonical injection, so that $\left.\varphi\right|_{V}=q$. Write $V=U \oplus \operatorname{rad}(q)$. We then have that $q_{r}:=$ $\left.\varphi\right|_{U}=\left.q\right|_{U}$ is nonsingular. Thus, with $U^{\perp}=\left\{x \in W \mid b_{\varphi}(x, U)=0\right\}$, we get that $W=U \oplus U^{\perp}$. Clearly, $\operatorname{rad}(q) \subset U^{\perp}$. We also have $U^{\perp}=U^{\prime} \oplus \operatorname{rad} \varphi$ with $\left.\varphi\right|_{U^{\prime}}$ nonsingular. We consider two cases.

First case: Suppose that $\operatorname{rad}(q) \subset \operatorname{rad}(\varphi)$. We choose any basis of $\operatorname{rad}(q)$ and extend it to a basis of $\operatorname{rad}(\varphi)$ such that, with respect to this basis, we get $\left.q\right|_{\operatorname{rad}(q)} \cong$ $\left\langle c_{1}, \cdots, c_{s}\right\rangle$ and $\left.\varphi\right|_{\operatorname{rad}(\varphi)} \cong\left\langle c_{1}, \cdots, c_{s^{\prime \prime}}\right\rangle$, where $c_{i} \in F$ and $0 \leq s=\operatorname{dim}(\operatorname{rad}(q)) \leq$ $s^{\prime \prime}=\operatorname{dim}(\operatorname{rad}(\varphi))$. (2) follows readily by putting $\tau=\left.\varphi\right|_{U^{\prime}}$ and by noting that $V$ $($ resp. $W)$ decomposes orthogonally into $V=U \oplus \operatorname{rad}(q)\left(\operatorname{resp} . \quad W=U \oplus U^{\prime} \oplus\right.$ $\operatorname{rad}(\varphi))$.

Second case: Suppose that $\operatorname{rad}(q) \not \subset \operatorname{rad}(\varphi)$. Let $\left\{e_{1}, \cdots, e_{s}\right\}$ be a basis of $\operatorname{rad}(q)$ such that $e_{1} \notin \operatorname{rad}(\varphi)$. With the same notation as before, we have that $\operatorname{rad}(q) \subset$ $U^{\perp}=U^{\prime} \oplus \operatorname{rad}(\varphi)$. Hence, there exists an $f_{1} \in U^{\prime}$ such that $b_{\varphi}\left(e_{1}, f_{1}\right)=\lambda_{1} \neq 0$. In particular, $f_{1}$ is linearly independent of $\left\{e_{1}, \cdots, e_{s}\right\}$. Put $e_{1}^{\prime}=\frac{1}{\lambda_{1}} e_{1}$ and, for $2 \leq i \leq s$, put $e_{i}^{\prime}=e_{i}-\frac{\lambda_{i}}{\lambda_{1}} e_{1}$, where $\lambda_{i}=b_{\varphi}\left(e_{i}, f_{1}\right)$. Then $\left\{e_{1}^{\prime}, \cdots, e_{s}^{\prime}\right\}$ is again a basis of $\operatorname{rad}(q)$, and furthermore $b_{\varphi}\left(e_{i}^{\prime}, f_{1}\right)=\delta_{i 1}$.

Let $\widetilde{U}=U \oplus F e_{1}^{\prime} \oplus F f_{1}$, let $c_{1}=q\left(e_{1}^{\prime}\right)=\varphi\left(e_{1}^{\prime}\right)$ and $d_{1}=\varphi\left(f_{1}\right)$. Then $\left.\varphi\right|_{\widetilde{U}} \cong$ $\left.\varphi\right|_{U} \perp\left[c_{1}, d_{1}\right]$. Let $\tilde{V}=V \oplus F f_{1}$ and let $\widetilde{q}=\left.\varphi\right|_{\tilde{V}}$. It follows readily that $\operatorname{rad}(\widetilde{q})$ has basis $\left\{e_{2}^{\prime}, \cdots, e_{s}^{\prime}\right\}$ and that $\widetilde{V}$ has an orthogonal decomposition $\widetilde{V}=\widetilde{U} \oplus \operatorname{rad}(\widetilde{q})$, where $\left.\widetilde{q}\right|_{\widetilde{U}}=\left.\varphi\right|_{\widetilde{U}}$ is nonsingular. In particular, $\operatorname{dim}(\operatorname{rad}(\widetilde{q}))<\operatorname{dim}(\operatorname{rad}(q))=s$. (2) follows now readily by induction on $s$ together with the first case.

Remark 3.2. The proof actually shows that $q_{r}$ can be any nonsingular form with the property that $q \cong q_{r} \perp \mathrm{ql}(q)$.

Corollary 3.3. Let $(q, V)$ be a quadratic form of type $(r, s)$ and let $(\varphi, W)$ be a nonsingular quadratic form of dimension $2 r+2 s$. The following are equivalent.

(1) There exists an injective isometry $t:(q, V) \rightarrow(\varphi, W)$.

(2) There exist a nonsingular form $q_{r}$ of dimension $2 r$ and $c_{j}, d_{j} \in F, 1 \leq j \leq$ $s$, such that for $\sigma=\left\langle c_{1}, \cdots, c_{s}\right\rangle$ and $\rho=\left[c_{1}, d_{1}\right] \perp \cdots \perp\left[c_{s}, d_{s}\right]$, we have $q \cong q_{r} \perp \sigma$ and $\varphi \cong q_{r} \perp \rho$.

Definition 3.4. Let $q$ and $\varphi$ be forms over $F$.

(i) $q$ is said to be a subform of $\varphi$ if there exists a form $\tau$ such that $\varphi \cong q \perp \tau$. We then write $q \subset \varphi$.

(ii) $q$ is said to be dominated by $\varphi$ if the equivalent conditions in Lemma 3.1 hold. We then write $q \prec \varphi$ and call $\varphi$ a dominant form of $q$. With the same 
notation as in Lemma 3.1, the form $\left.\varphi\right|_{U}$ is called the complement of $q$ in $\varphi$, and we write $\left.\varphi\right|_{U}=q_{\varphi}^{c}$.

(iii) $\varphi$ is said to be a nonsingular completion (n.s.c.) of $q$ if the equivalent conditions in Corollary 3.3 hold.

Lemma 3.5. Let $c_{i}, c_{i}^{\prime}, d_{i} \in F, 1 \leq i \leq n$, and suppose that $\left\langle c_{1}, \cdots, c_{n}\right\rangle \cong$ $\left\langle c_{1}^{\prime}, \cdots, c_{n}^{\prime}\right\rangle$. Then there exist $d_{1}^{\prime}, \cdots, d_{n}^{\prime} \in F$ such that $\left[c_{1}, d_{1}\right] \perp \cdots \perp\left[c_{n}, d_{n}\right] \cong$ $\left[c_{1}^{\prime}, d_{1}^{\prime}\right] \perp \cdots \perp\left[c_{n}^{\prime}, d_{n}^{\prime}\right]$.

Proof. This follows readily from the standard relations and the fact that by assumption, $\left\{c_{1}, \cdots, c_{n}\right\}$ and $\left\{c_{1}^{\prime}, \cdots, c_{n}^{\prime}\right\}$ span the same $F^{2}$-vector space.

Remark 3.6. (i) The notion of domination was introduced in [28, 31] using condition (2) in Lemma 3.1 as the definition, but under the somewhat more restrictive and less natural assumption that the $c_{i}$ are already given. If the dominating form is nonsingular (the most important case), then this notion of domination coincides with the one given here as follows immediately from Lemma 3.5 . In general, however, our notion is less restrictive. For example, let $F=K(X)$ be the rational function field in one variable over the field $K$. Then, according to our definition, the form $\langle 1, x\rangle$ is dominated by $\mathbb{H} \perp\langle 1+x\rangle$ as $\langle 1, x\rangle \cong\langle 1,1+x\rangle$, but not according to the definition in [28, 31] as $1+x \not \equiv 1, x \bmod F^{* 2}$.

Condition (2) is useful for practical purposes, e.g., when doing explicit computations with coefficients in a normalized form. However, condition (1) is more natural and conceptual.

(ii) A subform of another form is obviously dominated by this other form. The notions of subform and dominated form coincide if either the dominated form is nonsingular, or if both forms are totally singular.

(iii) The notion of a complement has also been defined by Knebusch [25], but only in the case where the dominant form is nonsingular. In this situation and with the above notation, Knebusch calls the complement $\left.\varphi\right|_{U}$ the polar of $\varphi$ with respect to the subspace $U$.

(iv) Assuming that $q \prec \varphi$ and with the same notation as in Lemma 3.1, we have that $q_{\varphi}^{c} \cong \tau \perp\left\langle c_{1}, \cdots, c_{s^{\prime \prime}}\right\rangle$. It is important to note that up to isometry, $q_{\varphi}^{c}$ is independent of the choice of the injective isometry $t$.

Indeed, suppose we have representations as in Lemma 3.1(ii) and other such representations

$$
\begin{gathered}
q \cong \widetilde{q_{r}} \perp\left\langle\widetilde{c_{1}}, \cdots, \widetilde{c_{s}}\right\rangle \\
\varphi \cong \widetilde{q_{r}} \perp \widetilde{\tau} \perp\left[\widetilde{c_{1}}, \widetilde{d_{1}}\right] \perp \cdots \perp\left[\widetilde{c_{s^{\prime}}}, \widetilde{d_{s^{\prime}}}\right] \perp\left\langle\widetilde{c_{s^{\prime}}+1}, \cdots, \widetilde{c_{s^{\prime \prime}}}\right\rangle .
\end{gathered}
$$

(Note that the values $s^{\prime} \leq s \leq s^{\prime \prime}$ and the dimensions of the forms and their radicals will necessarily be the same in both representations.) We have to show that

$$
\tau \perp\left\langle c_{1}, \cdots, c_{s^{\prime \prime}}\right\rangle \cong \widetilde{\tau} \perp\left\langle\widetilde{c_{1}}, \cdots, \widetilde{c_{s^{\prime \prime}}}\right\rangle .
$$

By comparing the quasi-linear parts, we easily obtain $\left\langle c_{1}, \cdots, c_{s}\right\rangle \cong\left\langle\widetilde{c_{1}}, \cdots, \widetilde{c_{s}}\right\rangle$ and $\left\langle c_{s^{\prime}+1}, \cdots, c_{s^{\prime \prime}}\right\rangle \cong\left\langle\widetilde{c_{s^{\prime}}+1}, \cdots, \widetilde{c_{s^{\prime \prime}}}\right\rangle$. Using $\langle c, c\rangle \sim\langle c\rangle$ we obtain $\left\langle c_{1}, \cdots, c_{s}\right\rangle \perp$ $\left\langle c_{s^{\prime}+1}, \cdots, c_{s^{\prime \prime}}\right\rangle \sim\left\langle c_{1}, \cdots, c_{s^{\prime \prime}}\right\rangle$ and thus, by comparing dimensions, $\left\langle c_{1}, \cdots, c_{s^{\prime \prime}}\right\rangle \cong$ $\left\langle\widetilde{c_{1}}, \cdots, \widetilde{c_{s^{\prime \prime}}}\right\rangle$. This implies

$$
\begin{aligned}
\varphi \perp\left\langle c_{1}, \cdots, c_{s^{\prime \prime}}\right\rangle & \sim q_{r} \perp \tau \perp\left\langle c_{1}, \cdots, \widetilde{c_{s^{\prime \prime}}}\right\rangle \\
& \left.\sim \varphi \perp\left\langle\widetilde{c_{1}}, \cdots, \widetilde{c_{s^{\prime \prime}}}\right\rangle, \widetilde{c_{s^{\prime \prime}}}\right\rangle, \\
& \sim \widetilde{q_{r}} \perp \bar{\tau} \perp\left\langle\overline{c_{1}}, \cdots,\right.
\end{aligned}
$$


and adding on the right-hand side in the first line $q \cong q_{r} \perp\left\langle c_{1}, \cdots, c_{s}\right\rangle$, and in the last line $q \cong \widetilde{q_{r}} \perp\left\langle\widetilde{c_{1}}, \cdots, \widetilde{c_{s}}\right\rangle$, then we obtain immediately $\tau \perp\left\langle c_{1}, \cdots, c_{s^{\prime \prime}}\right\rangle \sim \widetilde{\tau} \perp$

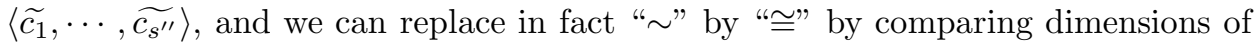
the forms and their radicals.

The following easy lemma is quite useful and will be used frequently.

Lemma 3.7. Let $q \prec \varphi$. Then $q \perp \varphi \sim q_{\varphi}^{c}$. In particular, if $\sigma$ is a totally singular form over $F$ and $\rho$ is a nonsingular completion of $\sigma$, then $\rho \perp \sigma \sim \sigma$.

Proof. This follows readily from the definitions of the complement and of a nonsingular completion, together with Remark 3.6(iv) and the fact that $[c, d] \perp\langle c\rangle \sim$ $\langle c\rangle \sim\langle c, c\rangle$.

Remark 3.8. If $\varphi$ is anisotropic and $q \prec \varphi$, then for another form $\gamma$ we have $\gamma \cong q_{\varphi}^{c}$ iff $q \perp \varphi \sim \gamma$ and $\gamma$ is anisotropic.

If we assume neither anisotropy nor nondefectiveness for $\varphi$, we need to invoke other hypotheses to get a similar result. Suppose that $q \prec \varphi^{\prime} \subset \varphi$ with $\varphi^{\prime}$ nonsingular. Let $\gamma$ be another form. Then $\gamma \cong q_{\varphi}^{c}$ iff $q \perp \varphi \sim \gamma, \operatorname{dim} \varphi=\operatorname{dim} \gamma+\operatorname{dim} q$, $\operatorname{dim} \operatorname{rad}(\gamma)=\operatorname{dim} \operatorname{rad}(\varphi)+\operatorname{dim} \operatorname{rad}(q)$. Indeed, we have $q \perp \varphi \sim q_{\varphi}^{c}$ by the above lemma, hence $\gamma \sim q_{\varphi}^{c}$, and the conditions on the dimensions plus the fact that $q$ is dominated by a nonsingular form inside $\varphi$ guarantee that $q_{\varphi}^{c}$ and $\gamma$ are of the same type.

Suppose that $\varphi$ and $\psi$ are nonsingular, $\sigma$ is totally singular, and $\rho$ is a nonsingular completion of $\sigma$. Then, in general, $\varphi \perp \sigma \cong \psi \perp \sigma$ does not imply $\varphi \perp \rho \cong \psi \perp \rho$. For example, suppose that $[a, b]$ is anisotropic. If we put $\varphi=\rho=[a, b], \psi=[0,0]$ and $\sigma=\langle a\rangle$, then $\rho$ is a nonsingular completion of $\sigma$ and we clearly have $\varphi \perp \sigma \cong$ $\psi \perp \sigma$. Now $\varphi \perp \rho \cong \mathbb{H} \perp \mathbb{H}$ is hyperbolic, but $\psi \perp \rho \cong \mathbb{H} \perp[a, b]$ isn't. However, we do have the following result which we will use in Section 5 .

Lemma 3.9. Let $\varphi, \psi$ be nonsingular, and let $\sigma$ be totally singular. Let $\rho$ be a fixed nonsingular completion of $\sigma$. If $\varphi \perp \sigma \cong \psi \perp \sigma$, then there exists a nonsingular completion $\rho^{\prime}$ of $\sigma$ such that $\varphi \perp \rho \cong \psi \perp \rho^{\prime}$.

Proof. Let $W$ (resp. $V$ ) be the underlying vector space of $\varphi \perp \sigma$ (resp. $\psi \perp \sigma$ ) with corresponding orthogonal decomposition $W=W_{\varphi} \oplus W_{\sigma}$ (resp. $V=V_{\psi} \oplus V_{\sigma}$ ). Let $W^{\prime} \supset W$ be the underlying vector space of $\varphi \perp \rho$ such that $\left.(\varphi \perp \rho)\right|_{W}=\varphi \perp \sigma$, and let $W^{\prime}=W_{\varphi} \oplus W_{\rho}$ be the corresponding orthogonal decomposition with $W_{\sigma} \subset W_{\rho}$. The isometry $\psi \perp \sigma \cong \varphi \perp \sigma$ yields an injective isometry $t:(\psi \perp \sigma, V) \rightarrow$ $\left(\varphi \perp \rho, W^{\prime}\right)$. The result now follows readily by noting that there is an orthogonal decomposition $W=t\left(V_{\psi}\right) \oplus t\left(V_{\sigma}\right)$ with $t\left(V_{\sigma}\right)=W_{\sigma}$ (an isometry maps radical onto radical) and $\left.(\varphi \perp \sigma)\right|_{t\left(V_{\psi}\right)} \cong \psi$, and by applying Corollary 3.3 together with Remark 3.2

Corollary 3.10. Let $\varphi, \psi$ be nonsingular, and let $\sigma, \tau$ be totally singular. Let $\rho$ be a fixed nonsingular completion of $\sigma$. If $\varphi \perp \sigma \perp \tau \cong \psi \perp \sigma \perp \tau$, then there exists a nonsingular completion $\rho^{\prime}$ of $\sigma$ such that $\varphi \perp \rho \perp \tau \cong \psi \perp \rho^{\prime} \perp \tau$.

Proof. Let $\eta$ be any n.s.c. of $\tau$. By Lemma 3.9 and Lemma 3.5 there exist n.s.c.'s $\rho^{\prime}$ of $\sigma$ and $\eta^{\prime}$ of $\tau$ such that $\varphi \perp \rho \perp \eta \cong \psi \perp \rho^{\prime} \perp \eta^{\prime}$. Adding $\tau$ on both sides and using $\eta \perp \tau \sim \eta^{\prime} \perp \tau \sim \tau$ (Lemma 3.7), we get $\varphi \perp \rho \perp \tau \sim \psi \perp \rho^{\prime} \perp \tau$. Since both forms are of the same type, we get $\varphi \perp \rho \perp \tau \cong \psi \perp \rho^{\prime} \perp \tau$. 
It is known in characteristic $\neq 2$ that if the orthogonal difference of two nonsingular forms of dimension $\geq m$ has Witt index $\geq m$, then these two forms have isometric subforms of dimension $m$. This can be generalized to characteristic 2 .

Proposition 3.11. Let $\varphi$ and $\psi$ be forms over $F$ and let $n(\varphi, \psi)=i_{\mathrm{ti}}(\varphi \perp \psi)-$ $\max \left\{i_{\mathrm{ql}}(\varphi), i_{\mathrm{ql}}(\psi)\right\}$.

(i) Let $m \leq \min \{\operatorname{dim} \varphi, \operatorname{dim} \psi, n(\varphi, \psi)\}$. Then there exists a form $q$ of dimension $m$ with $q \prec \varphi, q \prec \psi$.

(ii) Let $q$ be a form with $q \prec \varphi, q \prec \psi$. Then $\operatorname{dim} q \leq n(\varphi, \psi)$.

(iii) $\min \{\operatorname{dim} \varphi, \operatorname{dim} \psi, n(\varphi, \psi)\}$ is the maximal dimension of forms $q$ with $q \prec$ $\varphi, q \prec \psi$.

Proof. (iii) follows readily from (i) and (ii).

Proof of (i). First, we reduce the statement to the case where one of the two forms is nondefective. Write $\varphi \cong \varphi_{\text {nd }} \perp r \times\langle 0\rangle$ and $\psi \cong \psi_{\text {nd }} \perp s \times\langle 0\rangle$ with $\varphi_{\text {nd }}, \psi_{\text {nd }}$ nondefective, and assume $r \leq s$. Put $\varphi^{\prime}=\varphi_{\text {nd }}$ and $\psi^{\prime}=\psi_{\text {nd }} \perp(s-r) \times\langle 0\rangle$. We have $\max \left\{i_{\mathrm{ql}}(\varphi), i_{\mathrm{ql}}(\psi)\right\}=s, \max \left\{i_{\mathrm{ql}}\left(\varphi^{\prime}\right), i_{\mathrm{ql}}\left(\psi^{\prime}\right)\right\}=s-r$ and $i_{\mathrm{ti}}(\varphi \perp \psi)=$ $i_{\mathrm{ti}}\left(\varphi^{\prime} \perp \psi^{\prime}\right)+2 r$. Hence,

$$
m-r \leq \min \left\{\operatorname{dim} \varphi^{\prime}, \operatorname{dim} \psi^{\prime}, n\left(\varphi^{\prime}, \psi^{\prime}\right)\right\} .
$$

Assuming our result to be true in the case where one of the forms is nondefective, we conclude that there exists a form $q^{\prime}$ with $\operatorname{dim} q^{\prime}=m-r$ such that $q^{\prime} \prec \varphi^{\prime}$ and $q^{\prime} \prec \psi^{\prime}$. The form $q=q^{\prime} \perp r \times\langle 0\rangle$ is the desired form.

So, from now on let us assume that $\varphi$ is nondefective and that $\psi \cong \psi_{\text {nd }} \perp s \times\langle 0\rangle$ with $\psi_{\text {nd }}$ nondefective. We then have

$$
i_{\mathrm{ti}}(\varphi \perp \psi)-\max \left\{i_{\mathrm{ql}}(\varphi), i_{\mathrm{ql}}(\psi)\right\}=i_{\mathrm{ti}}(\varphi \perp \psi)-s=i_{\mathrm{ti}}\left(\varphi \perp \psi_{\mathrm{nd}}\right) .
$$

We use induction on $m$. If $m=0$ there is nothing to show. So suppose $m>0$.

Assume that $\varphi$ and $\psi$ have a common nonsingular subform $\alpha$ with $\operatorname{dim} \alpha>0$. Then $\varphi \cong \varphi^{\prime} \perp \alpha, \psi \cong \psi^{\prime} \perp \alpha$ and $i_{\mathrm{ql}}\left(\psi^{\prime}\right)=i_{\mathrm{ql}}(\psi)=s$. We now use the induction hypothesis applied to $\varphi^{\prime} \perp \psi^{\prime}$. Indeed, Witt cancellation yields

$$
i_{\mathrm{ti}}\left(\varphi^{\prime} \perp \psi^{\prime}\right)-s \geq m-\operatorname{dim} \alpha,
$$

and $\min \left\{\operatorname{dim} \varphi^{\prime}, \operatorname{dim} \psi^{\prime}, m-\operatorname{dim} \alpha\right\}=m-\operatorname{dim} \alpha$ then shows the existence of a form $q^{\prime}$ of dimension $m-\operatorname{dim} \alpha$ with $q^{\prime} \prec \varphi^{\prime}, q^{\prime} \prec \psi^{\prime}$. Thus, $q \cong q^{\prime} \perp \alpha$ is the desired form.

Therefore, we may assume that $\varphi$ and $\psi$ do not contain a common nonsingular subform. Applied to the form $\mathbb{H}$, this implies in particular that $\varphi$ or $\psi_{\text {nd }}$ is anisotropic.

Suppose that $\varphi$ is isotropic. Then, by the above, we may assume that $\psi_{\text {nd }}$ is anisotropic. The nondefectiveness implies that $\varphi \cong \mathbb{H} \perp \varphi^{\prime}$ and we get

$$
i_{\mathrm{ti}}\left(\varphi^{\prime} \perp \psi\right)-s \geq m-1 .
$$

By induction, there exists a totally singular form $q^{\prime}$ such that $\operatorname{dim} q^{\prime}=m^{\prime}=$ $\min \left\{\operatorname{dim} \varphi^{\prime}, \operatorname{dim} \psi, m-1\right\}$ and $q^{\prime} \prec \varphi^{\prime}, q^{\prime} \prec \psi$. Since by assumption $m \leq \operatorname{dim} \varphi$, $\operatorname{dim} \psi$ and $\operatorname{dim} \varphi^{\prime}=\operatorname{dim} \varphi-2$, there are two cases: $m^{\prime}=m-1$ (if $\operatorname{dim} \varphi \geq m+1$ ) and $m^{\prime}=m-2$ (if $\operatorname{dim} \varphi=m$ ).

Suppose $m^{\prime}=m-1$. In this case, we can write $q^{\prime} \cong \beta \perp \gamma$ with $\beta, \gamma$ totally singular, $\psi \cong \beta_{1} \perp \gamma \perp \delta$ with $\beta_{1}$ a nonsingular completion of $\beta$ and some form 
$\delta$. Now $\operatorname{dim} \psi>m^{\prime}$ implies that $\operatorname{dim} \beta_{1}+\operatorname{dim} \delta \geq 1$. If $\operatorname{dim} \delta \geq 1$, then for any $a \in D_{F}(\delta)$ we have $\langle a\rangle \prec \mathbb{H}$ as $D_{F}(\mathbb{H})=F$, and hence $q^{\prime} \perp\langle a\rangle \prec \varphi^{\prime} \perp \mathbb{H} \cong \varphi$ and $q^{\prime} \perp\langle a\rangle \prec \psi$. If $\operatorname{dim} \beta_{1} \geq 1$, then there exist $b, d \in F$ such that $\langle b\rangle \subset \beta$, and $[b, d] \subset \beta_{1}$. Using the fact that $[b, d] \perp\langle b\rangle \cong \mathbb{H} \perp\langle b\rangle \prec \varphi$, we now conclude that $[b, d] \subset \varphi$ and $[b, d] \subset \psi$, a contradiction to our hypothesis of the nonexistence of common nonsingular subforms.

If $m^{\prime}=\operatorname{dim} \varphi^{\prime}=m-2$, then $\operatorname{dim} \psi_{\text {nd }}+m-1>\operatorname{dim}\left(\varphi^{\prime} \perp \psi_{\text {nd }}\right)$, hence, for dimension reasons, the underlying subspace of $\psi_{\text {nd }}$ inside the underlying vector space of $\varphi^{\prime} \perp \psi_{\text {nd }}$ intersects with the totally isotropic subspace of dimension $i_{\mathrm{ti}}\left(\varphi^{\prime} \perp \psi_{\mathrm{nd}}\right)=i_{\mathrm{ti}}\left(\varphi^{\prime} \perp \psi\right)-s \geq m-1$, which in turn yields that $\psi_{\text {nd }}$ is isotropic, which by nondefectiveness implies $\mathbb{H} \subset \psi_{\text {nd }}$, again a contradiction because by assumption $\mathbb{H} \subset \varphi$ as well.

The same reasoning can be applied when $\psi_{\text {nd }}$ is isotropic. In this case we write $\psi \cong \mathbb{H} \perp \psi^{\prime}$, we simply replace $\varphi^{\prime}$ by $\psi^{\prime}, \psi$ and $\psi_{\text {nd }}$ by $\varphi$ in the above argument and we note that $i_{\mathrm{ti}}\left(\psi^{\prime} \perp \varphi\right) \geq m-1+s \geq m-1$.

Thus, we may finally assume that $\varphi$ and $\psi_{\text {nd }}$ are anisotropic, and that $\varphi$ and $\psi$ do not contain common nonsingular subforms.

Let $V$ and $W$ be the underlying vector spaces of $\varphi$ and $\psi$, respectively. By induction hypothesis, there exist subspaces $V^{\prime} \subset V$ and $W^{\prime} \subset W$ with $\operatorname{dim} V^{\prime}=$ $\operatorname{dim} W^{\prime}=m-1$ such that $\left.\left.q^{\prime} \cong \varphi\right|_{V^{\prime}} \cong \psi\right|_{W^{\prime}}$ with $q^{\prime}$ totally singular. Thus, there exist orthogonal bases $\left\{e_{1}, \cdots, e_{m-1}\right\}$ and $\left\{f_{1}, \cdots, f_{m-1}\right\}$ of $V^{\prime}$ and $W^{\prime}$, respectively, with $\varphi\left(e_{i}\right)=\psi\left(f_{i}\right)=c_{i} \in F^{*}$. In particular, $\left\{e_{1}+f_{1}, \cdots, e_{m-1}+\right.$ $\left.f_{m-1}\right\}$ is a basis of a totally isotropic subspace $U$ of $(\varphi \perp \psi, V \oplus W)$.

Let $W=W_{\text {nd }} \oplus W_{0}$ with $\left.\psi\right|_{W_{\text {nd }}} \cong \psi_{\text {nd }}$ and $W_{0} \subset \operatorname{rad}(\psi),\left.\psi\right|_{W_{0}} \cong s \times\langle 0\rangle$. We fix a basis $\left\{g_{1}, \cdots, g_{s}\right\}$ of $W_{0}$. The anisotropy of $\varphi$ implies that $q^{\prime}$ is anisotropic, hence $W^{\prime} \cap W_{0}=\{0\}$. In particular, we have a direct sum of subvector spaces $V^{\prime} \oplus W^{\prime} \oplus W_{0}$ of $V \oplus W$ and therefore also a direct sum $U \oplus W_{0}$. Clearly, $U \oplus W_{0}$ is a totally isotropic subspace of $(\varphi \perp \psi, V \oplus W)$ of dimension $m-1+s$. This totally isotropic subspace is contained in a maximal totally isotropic subspace of $(\varphi \perp \psi, V \oplus W)$ which, by assumption, is of dimension $\geq m+s$.

Hence, there exist $v \in V$ and $w \in W$ such that $v+w \notin U \oplus W_{0}$ and such that $\left\{e_{1}+f_{1}, \cdots, e_{m-1}+f_{m-1}, g_{1}, \cdots, g_{s}, v+w\right\}$ is a basis for a totally isotropic subspace of dimension $m+s$. In particular, $\varphi(v)+\psi(w)=0$. Suppose that $\varphi(v)=0$. The anisotropy of $\varphi$ implies that $v=0$. In particular, $w \neq 0$ and $\psi(w)=0$, and we have that $\left\{g_{1}, \cdots, g_{s}, w\right\}$ is in fact a totally isotropic subspace of $(\psi, W)$ of dimension $s+1$. But we assumed that $\psi \cong \psi_{\text {nd }} \perp s \times\langle 0\rangle$ with $\psi_{\text {nd }}$ anisotropic, which, in particular, yields that $i_{\mathrm{ti}}(\psi)=s<s+1$, a contradiction. Therefore, $v \neq 0$ and the anisotropy of $\varphi$ implies $a=\varphi(v)=\psi(w) \neq 0$. Note also that $0=b_{\varphi \perp \psi}\left(e_{i}+f_{i}, v+w\right)=b_{\varphi}\left(e_{i}, v\right)+b_{\psi}\left(f_{i}, w\right)$ and therefore, $b_{\varphi}\left(e_{i}, v\right)=b_{\psi}\left(f_{i}, w\right)$.

Suppose $v \in V^{\prime}$. Then there exists $w^{\prime} \in W^{\prime}$ with $a=\varphi(v)=\psi\left(w^{\prime}\right)=\psi(w)$. Also, $b_{\varphi}\left(e_{i}, v\right)=0$ for all $i$ and hence $b_{\psi}\left(f_{i}, w\right)=0$ for all $i$ and thus $b_{\psi}\left(w^{\prime}, w\right)=$ 0 . We also clearly have $b_{\psi}\left(g_{i}, w\right)=0$ for all $g_{i}$. Suppose now that $w \notin W^{\prime} \oplus$ $W_{0}$. The above then shows that the quadratic subspace of $(\psi, W)$ spanned by $\left\{w, w^{\prime}, g_{1}, \cdots, g_{s}\right\}$ is isometric to $\langle a, a\rangle \perp s \times\langle 0\rangle \cong\langle a\rangle \perp(s+1) \times\langle 0\rangle$, again a contradiction to the fact that $i_{\mathrm{ti}}(\psi)=s$. Hence, $w \in W^{\prime} \oplus W_{0}$. Let $v=\sum_{i=1}^{m-1} a_{i} e_{i}$ and $w=\sum_{i=1}^{m-1} b_{i} f_{i}+\sum_{j=1}^{s} c_{j} g_{j}, a_{i}, b_{i}, c_{i} \in F$. Then $\sum_{i=1}^{m-1} a_{i}^{2} \varphi\left(e_{i}\right)=\varphi(v)=$ $\psi(w)=\sum_{i=1}^{m-1} b_{i}^{2} \psi\left(f_{i}\right)$. Now $\varphi\left(e_{i}\right)=\psi\left(f_{i}\right)=c_{i}$ and the fact that $\left.\varphi\right|_{V^{\prime}}$ and $\left.\psi\right|_{W^{\prime}}$ are anisotropic totally singular implies that the $c_{i}$ are $F^{2}$-linearly independent, 
hence $a_{i}^{2}=b_{i}^{2}$ for all $1 \leq i \leq m-1$ and thus $a_{i}=b_{i}$. In particular, $v+w=$ $\sum_{i=1}^{m-1} a_{i}\left(e_{i}+f_{i}\right)+\sum_{j=1}^{s} c_{j} g_{j} \in U \oplus W_{0}$, a contradiction.

We conclude that $v \notin V^{\prime}$. A similar argument as above shows that $w \notin W^{\prime}$. Let $\tilde{V}=V^{\prime} \oplus F v$ and $\tilde{W}=W^{\prime} \oplus F w$. One verifies readily that $\left.\left.\left.\varphi\right|_{\tilde{V}} \cong \psi\right|_{\tilde{W}} \cdot q \cong \varphi\right|_{\tilde{V}}$ is then the desired form of dimension $m$.

In the proof of part (ii), we will need the following.

Lemma 3.12. Let $\varphi$ and $q$ be forms with $q \prec \varphi$ and $\mathrm{ql}(q) \cong q^{\prime} \perp(t \times\langle 0\rangle)$. Then there exist a form $\varphi^{\prime}$ and a nonsingular form $q_{0}$ such that $\varphi \cong \varphi^{\prime} \perp\left(r_{1} \times \mathbb{H}\right) \perp$ $\left(r_{2} \times\langle 0\rangle\right)$ with $q \cong q_{0} \perp \mathrm{ql}(q), q_{0} \perp q^{\prime} \prec \varphi^{\prime}$ and $t=r_{1}+r_{2}$.

Proof. By Lemma 3.1, it suffices to consider the case where $q$ is totally singular, i.e., $q=\mathrm{ql}(q)$. Suppose that $\operatorname{dim} q=k$. Then $q \prec \varphi$ implies that there exist an integer $\ell, 0 \leq \ell \leq k, c_{1}, \cdots, c_{k} \in F, a_{1}, \cdots, a_{\ell} \in F$, and a form $\alpha$ such that $q \cong\left\langle c_{1}, \cdots, c_{k}\right\rangle$ and

$$
\varphi \cong \alpha \perp\left[a_{1}, c_{1}\right] \perp \cdots \perp\left[a_{\ell}, c_{\ell}\right] \perp\left\langle c_{\ell+1}, \cdots, c_{k}\right\rangle .
$$

Using the standard relations, we can rewrite $\left[a_{1}, c_{1}\right] \perp \cdots \perp\left[a_{\ell}, c_{\ell}\right] \perp\left\langle c_{\ell+1}, \cdots, c_{k}\right\rangle$ in such a way that we may assume that there exist $r_{1}, r_{2} \geq 0$ with $\ell \geq r_{1}, k-\ell \geq r_{2}$ such that

$$
c_{1}=\cdots=c_{r_{1}}=c_{k-r_{2}+1}=\cdots=c_{k}=0 .
$$

Putting $q^{\prime \prime}=\left\langle c_{r_{1}+1}, \cdots, c_{k-r_{2}}\right\rangle$, it follows that we can write $q \cong q^{\prime \prime} \perp(t \times\langle 0\rangle)$,

$$
\varphi \cong \varphi^{\prime} \perp\left(r_{1} \times \mathbb{H}\right) \perp\left(r_{2} \times\langle 0\rangle\right)
$$

with $q^{\prime \prime} \prec \varphi^{\prime}$ and $t=r_{1}+r_{2}$. Now $q^{\prime} \cong q^{\prime \prime}$ follows readily from Proposition 8.1 (i) (or Lemma 2.6).

Proof of (ii). We may assume that $i_{\mathrm{ql}}(\psi)=s \geq r=i_{\mathrm{ql}}(\varphi)$. Suppose there exists a form $q$ with $q \prec \varphi, q \prec \psi$ and $\operatorname{dim} q>n(\varphi, \psi)=i_{\mathrm{ti}}(\varphi \perp \psi)-s$.

First, we reduce to the case where $q$ is totally singular. If $q$ is not totally singular, then we can write $q \cong \rho \perp \sigma$ with $\rho$ nonsingular of dimension $\geq 2$ and $\sigma=\operatorname{ql}(q)$. Now $q \prec \varphi, q \prec \psi$ and the nonsingularity of $\rho$ readily imply that $\varphi \cong \rho \perp \varphi^{\prime}, \psi \cong \rho \perp \psi^{\prime}$ for certain forms $\varphi^{\prime}, \psi^{\prime}$ with $\sigma \prec \varphi^{\prime}, \sigma \prec \psi^{\prime}$. We have $\rho \perp \rho \cong(\operatorname{dim} \rho) \times \mathbb{H}$. Thus,

$$
\operatorname{dim} \sigma=\operatorname{dim} q-\operatorname{dim} \rho>i_{\mathrm{ti}}(\varphi \perp \psi)-s-\operatorname{dim} \rho=i_{\mathrm{ti}}\left(\varphi^{\prime} \perp \psi^{\prime}\right)-s .
$$

We also have $i_{\mathrm{ql}}\left(\varphi^{\prime}\right)=i_{\mathrm{ql}}(\varphi)=r \leq s=i_{\mathrm{ql}}(\psi)=i_{\mathrm{ql}}\left(\psi^{\prime}\right)$ as the quasi-linear parts of $\varphi($ resp. $\psi)$ and $\varphi^{\prime}\left(\right.$ resp. $\left.\psi^{\prime}\right)$ are the same.

Hence, we get $\sigma \prec \varphi^{\prime}, \sigma \prec \psi^{\prime}$, and $\operatorname{dim} \sigma>n\left(\varphi^{\prime}, \psi^{\prime}\right)$ with $\sigma$ totally singular. Thus, to get a contradiction, it suffices to consider the case where $q$ is totally singular.

So let us assume that $q$ is totally singular and write $q \cong q^{\prime} \perp(t \times\langle 0\rangle)$ with $q^{\prime}$ anisotropic. By Lemma 3.12,

$$
\begin{aligned}
& \varphi \cong \varphi^{\prime} \perp\left(r_{1} \times \mathbb{H}\right) \perp\left(r_{2} \times\langle 0\rangle\right), \\
& \psi \cong \psi^{\prime} \perp\left(s_{1} \times \mathbb{H}\right) \perp\left(s_{2} \times\langle 0\rangle\right)
\end{aligned}
$$

with $q^{\prime} \prec \varphi^{\prime}, q^{\prime} \prec \psi^{\prime}$, and $t=r_{1}+r_{2}=s_{1}+s_{2}$. In fact, the anisotropy of $q^{\prime}$ implies that $q^{\prime}$ is dominated by the nondefective parts of $\varphi$ and $\psi$, i.e., we have $q^{\prime} \prec \varphi_{\text {nd }}^{\prime}, \psi_{\text {nd }}^{\prime}$. 
By assumption, $\operatorname{dim} q^{\prime}+t=\operatorname{dim} q>i_{\mathrm{ti}}(\varphi \perp \psi)-s$. We have

$$
i_{\mathrm{ti}}(\varphi \perp \psi)=i_{\mathrm{ti}}\left(\varphi^{\prime} \perp \psi^{\prime}\right)+r_{1}+r_{2}+s_{1}+s_{2}=i_{\mathrm{ti}}\left(\varphi^{\prime} \perp \psi^{\prime}\right)+2 t .
$$

Also, $i_{\mathrm{ql}}\left(\varphi^{\prime}\right)=r-r_{2}, i_{\mathrm{ql}}\left(\psi^{\prime}\right)=s-s_{2}$. Therefore,

$$
i_{\mathrm{ti}}\left(\varphi^{\prime} \perp \psi^{\prime}\right)=i_{\mathrm{ti}}\left(\varphi_{\text {nd }}^{\prime} \perp \psi_{\text {nd }}^{\prime}\right)+\left(r-r_{2}\right)+\left(s-s_{2}\right) \text {. }
$$

Thus,

$$
\begin{aligned}
\operatorname{dim} q^{\prime}>i_{\mathrm{ti}} & \left(\varphi_{\mathrm{nd}}^{\prime} \perp \psi_{\mathrm{nd}}^{\prime}\right)+t+\left(r-r_{2}\right)+\left(s-s_{2}\right)-s \\
& =i_{\mathrm{ti}}\left(\varphi_{\mathrm{nd}}^{\prime} \perp \psi_{\mathrm{nd}}^{\prime}\right)+s_{1}+s_{2}+\left(r-r_{2}\right)-s_{2} \\
& =i_{\mathrm{ti}}\left(\varphi_{\mathrm{nd}}^{\prime} \perp \psi_{\mathrm{nd}}^{\prime}\right)+s_{1}+\left(r-r_{2}\right) \\
& \geq i_{\mathrm{ti}}\left(\varphi_{\mathrm{nd}}^{\prime} \perp \psi_{\mathrm{nd}}^{\prime}\right) .
\end{aligned}
$$

Now $q^{\prime} \perp q^{\prime} \cong q^{\prime} \perp\left(\operatorname{dim} q^{\prime} \times\langle 0\rangle\right) \prec \varphi_{\text {nd }}^{\prime} \perp \psi_{\text {nd }}^{\prime}$. Hence $i_{\text {ti }}\left(\varphi_{\text {nd }}^{\prime} \perp \psi_{\text {nd }}^{\prime}\right) \geq \operatorname{dim} q^{\prime}$, a contradiction.

Corollary 3.13. Let $\varphi$ and $\psi$ be nondefective forms and let $q$ be a form of maximal dimension such that $q \prec \varphi, q \prec \psi$. Then $\operatorname{dim} q=\min \left\{\operatorname{dim} \varphi, \operatorname{dim} \psi, i_{\mathrm{ti}}(\varphi \perp \psi)\right\}$ and $q$ is nondefective.

Proof. The statement about $\operatorname{dim} q$ follows immediately from the previous proposition by noting that nondefectiveness of $\varphi, \psi$ implies $n(\varphi, \psi)=i_{\mathrm{ti}}(\varphi \perp \psi)$.

Suppose that $\eta \prec \varphi, \eta \prec \psi$ and that $\eta$ is defective, say, $\eta \cong \eta^{\prime} \perp(t \times\langle 0\rangle)$ with $\eta^{\prime}$ nondefective and $t>0$. Lemma 3.12 together with the fact that $\varphi, \psi$ are nondefective imply that we can write $\varphi \cong \varphi^{\prime} \perp(t \times \mathbb{H}), \psi \cong \psi^{\prime} \perp(t \times \mathbb{H})$ with $\eta^{\prime} \prec \varphi^{\prime}, \eta^{\prime} \prec \psi^{\prime}$. For $\tilde{\eta}=\eta^{\prime} \perp(t \times \mathbb{H})$, we thus get $\tilde{\eta} \prec \varphi, \tilde{\eta} \prec \psi$ and $\operatorname{dim} \tilde{\eta}=\operatorname{dim} \eta+t>\operatorname{dim} \eta$. This implies the nondefectiveness of $q$.

\section{Further NOtions AND RESUlts NEEDED IN PROOFS}

4.1. Pfister forms and quasi-Pfister forms. Similar to the case of characteristic $\neq 2$, one can construct the Witt ring $W F$ of nondegenerate symmetric bilinear forms over $F$ and the Witt group $W_{q} F$ of nonsingular quadratic forms over $F$. $W_{q} F$ can be considered in a natural way as a $W F$-module by the operation $b \otimes q(x \otimes y)=$ $b(x, x) q(y)$.

An $n$-fold bilinear Pfister form is a form of type $b=\left\langle 1, a_{1}\right\rangle_{b} \otimes \cdots \otimes\left\langle 1, a_{n}\right\rangle_{b}$ for some $a_{i} \in F^{*}$; we write $\left\langle\left\langle a_{1}, \cdots, a_{n}\right\rangle_{b}\right.$ for short. If $q(X)=b(X, X)$ is the corresponding totally singular (hence quasi-linear) quadratic form, we write $q=\left\langle\left\langle a_{1}, \cdots, a_{n}\right\rangle\right\rangle$ and call it an $n$-fold quasi-Pfister form. An $(n+1)$-fold Pfister form is then a nonsingular quadratic form of type $\left\langle\left\langle a_{1}, \cdots, a_{n}\right\rangle\right\rangle_{b} \otimes[1, b]$ for some $a_{i} \in F^{*}, b \in F$. This Pfister form is then said to be of degree $n+1$ and we write $\left\langle\left\langle a_{1}, \cdots, a_{n} ; b\right]\right]$ for short. $\langle\langle b]]=[1, b]$ is thus a 1 -fold Pfister form. The set of forms isometric (resp. similar) to $n$-fold Pfister forms will be denoted by $P_{n} F$ (resp. $G P_{n} F$ ). We denote the $W F$-submodule of $W_{q} F$ generated by $n$-fold Pfister forms by $I^{n} W_{q} F$, and we obtain a filtration $W_{q} F=I W_{q} F \supset I^{2} W_{q} F \supset \cdots$.

Note that this definition of degree has also been given in [29, Sect. 6]. It differs from the one given in [6], 7], but ours is in closer analogy to the characteristic $\neq 2$ case, where $n$-fold Pfister forms are of degree $n$ and dimension $2^{n}$ just as according to our definition in characteristic 2 (and not $2^{n+1}$ as in the definition in [11]).

As is the case in characteristic $\neq 2, x \in F^{*}$ is represented by a Pfister form $\pi$ if and only if $x \pi \cong \pi$. This property is commonly referred to as $\pi$ being round. The same holds for quasi-Pfister forms (cf. also [35, page 38]). In fact, anisotropic quasi-Pfister forms can be characterized in this way as was noted by Knebusch 
[25]. For completeness' sake we will sketch a proof of these facts in Section 8 (see Proposition 8.5).

4.2. Function fields of quadratic forms and splitting towers. Suppose the quadratic form $q$ (which is supposed to be nonzero) is given by the polynomial $q(X, Y, Z)=\sum_{i=1}^{r}\left(a_{i} X_{i}^{2}+X_{i} Y_{i}+b_{i} Y_{i}^{2}\right)+\sum_{j=1}^{s} c_{j} Z_{j}^{2}$. Then the corresponding polynomial $q(X, Y, Z)$ is reducible iff the nondefective part $q_{\text {nd }}$ of $q$ is either of type $(0,1)$ or of type $(1,0)$ and $q_{\text {nd }} \cong \mathbb{H}$. It is absolutely irreducible iff $q$ is of type $(r, s)$ with $r \geq 1$ and $\operatorname{dim} q_{\text {nd }} \geq 3$ (see, e.g., [1]). Suppose that $q(X, Y, Z)$ is irreducible. Then the function field $F(q)$ of $q$ is defined to be the field of fractions of the quotient ring $F[X, Y, Z] /(q(X, Y, Z))$. To avoid case distinctions, we put $F(q)=F$ if $q(X, Y, Z)$ is reducible or $\operatorname{dim} q=0$. We have that $F(q) / F$ is purely transcendental iff $q_{\text {nd }}$ is isotropic. If $q(X, Y, Z)$ is absolutely irreducible and $K / F$ is any field extension, then the free compositum $K \cdot F(q)$ can be identified with $K(q)$, the quotient field of the integral domain $K[X, Y, Z] /(q(X, Y, Z))$.

Suppose $q$ is defective, say, $q \cong q_{\text {nd }} \perp m \times\langle 0\rangle$ with $q_{\text {nd }}$ the nondefective part of $q$, then $F(q)$ is a purely transcendental extension of $F\left(q_{\mathrm{nd}}\right)$ of transcendence degree $m$.

Of particular importance in this paper will be the isotropy behaviour of a quadratic form over the function field of another quadratic form. Since an anisotropic form stays anisotropic over purely transcendental extensions and by the previous remarks, this isotropy question is only of interest when both forms are anisotropic. The following lemma has been shown in [28, Cor. 3.1] and will be used frequently.

Lemma 4.1. Let $\varphi, \psi$ be anisotropic forms over $F$. Suppose that $\psi$ is not totally singular. Then $\mathrm{ql}(\varphi)_{F(\psi)}$ is anisotropic and is the quasi-linear part of $\left(\varphi_{F(\psi)}\right)_{\text {an }}$. In particular, if $\varphi$ is of type $(r, s)$, then $\left(\varphi_{F(\psi)}\right)$ an is of type $\left(r^{\prime}, s\right)$ with $r^{\prime} \leq r$.

Of utmost importance are the following four results. The first part of it will be referred to later on as the domination theorem, the last part is an analogue in characteristic 2 of the Arason-Pfister Hauptsatz and will be referred to as such.

Theorem 4.2. $\quad$ (i) Let $\varphi, \psi$ be forms over $F$ with $\varphi$ anisotropic and $\psi$ nondefective. If $\varphi_{F(\psi)}$ is hyperbolic, then ab $\psi \prec \varphi$ for each $a \in D_{F}(\varphi)$, $b \in D_{F}(\psi)$.

(ii) Let $\varphi$ be an anisotropic nonsingular form. Then $\varphi$ is similar (isometric) to a Pfister form if and only if $\varphi_{F(\varphi)}$ is hyperbolic (and $1 \in D_{F}(\varphi)$ ).

(iii) Let $\psi$ be anisotropic nonsingular, and let $\varphi$ be an anisotropic Pfister form. Then $\psi_{F(\varphi)}$ is hyperbolic if and only if there exist $a_{1}, \cdots, a_{m} \in F^{*}$ such that $\psi \cong a_{1} \varphi \perp \cdots \perp a_{m} \varphi$.

(iv) Let $q$ be a nonzero anisotropic form in $I^{n} W_{q} F$. Then $\operatorname{dim} q \geq 2^{n}$. If $\operatorname{dim} q=2^{n}$, then $q$ is similar to an $n$-fold Pfister form.

Remark 4.3. The corresponding version of the domination theorem in characteristic $\neq 2$ is an immediate consequence of the Cassels-Pfister subform theorem and was first explicitly stated independently by Wadsworth [38], Arason [3] and Knebusch [23]. The characteristic 2 version of the Cassels-Pfister subform theorem was first proved by Amer (cf. [35. pp. 17-18]). This subform theorem plus the norm theorem in characteristic 2 by Baeza [12] immediately yield the above domination theorem. See also [28], [31], where the above domination theorem has been stated perhaps for the first time explicitly. 
The characterization of forms similar (isometric) to Pfister forms is also an immediate consequence of the norm theorem [12] together with the characterization of Pfister forms as being the anisotropic (strictly) multiplicative forms; cf. [35] p. 38].

Part (iii) is a direct consequence of (i) and (ii).

The Arason-Pfister Hauptsatz in characteristic $\neq 2$ was shown in 5 and later in characteristic 2 in [10]. It is a straightforward consequence of the first two results.

The next important theorem which we will use later on has been shown in characteristic $\neq 2$ in 16. The characteristic 2 version below has been shown in 18 . Before that, a somewhat weaker version has been proved in 31 .

Theorem 4.4. Let $\varphi$ and $\psi$ be anisotropic forms over $F$ such that, for some positive integer $n$, one has $\operatorname{dim} \varphi \leq 2^{n}<\operatorname{dim} \psi$. Then $\varphi_{F(\psi)}$ is anisotropic.

In characteristic $\neq 2$, the foundations of a theory of generic splitting of quadratic forms, based on splitting towers as defined below, have been laid out by Knebusch 22, 23. Most of this generic theory goes through also in characteristic 2 for nonsingular forms, based on results in [10, 12]. The notion of generic splitting has recently been extended to forms which are possibly singular in a forthcoming book by Knebusch [25]. A theory of standard splitting towers for arbitrary forms in characteristic 2 has been developed in 29 .

The standard splitting tower of $q$ is defined to be the following sequence of field extensions of $F$. We put $F_{0}=F$ and $q_{0}=q_{\text {an }}$, the anisotropic part of $q$. For $n \geq 1$, we put $F_{n}=F_{n-1}\left(q_{n-1}\right)$ and $q_{n}=\left(\left(q_{n-1}\right)_{F_{n}}\right)$ an. The (standard) height $h(q)$ of $q$ is the smallest integer $r$ with $\operatorname{dim} q_{r} \leq 1$. Let $h=h(q)$ be the height of $q$. The form $q_{i}, 0 \leq i \leq h$ is called the $i$-th kernel form. If $h \geq 1$, then we call $F_{h-1}$ the leading field of $q$, and $q_{h-1}$ its leading form. Suppose that $q$ is anisotropic of type $(r, s)$ with $r \geq 1$. By the previous lemma, there exists an $i, 1 \leq i \leq h(q)$, such that, for $1 \leq j \leq h(q)$ one has that $q_{j}$ is of type $\left(r_{j}, s\right)$ with $r_{j}<r_{j-1}$ if $j \leq i$, and $r_{j}=0$ for $j \geq i$ (see also [29]). The value $i$ will be called the nondefective height of $q$ as it is the largest $i$ such that the quasi-linear part $\mathrm{ql}(q)$ of $q$ stays anisotropic over $F_{i}$, i.e., such that $q_{F_{i}}$ stays nondefective, and we write $h_{\text {nd }}(q)=i$. In Knebusch's generic theory for quadratic forms [25, it is shown that the standard splitting tower up to $F_{h_{\text {nd }}}$ is a generic splitting tower according to his definition of genericity, and his definition of height corresponds to what we call nondefective height. Some results on the standard splitting tower and the height of singular forms will be shown in Section 8 In particular, we will show

Theorem 4.5. Let $q$ be an anisotropic form of standard height $h$. Let $F=K_{0} \subset$ $K_{1} \subset \cdots \subset K_{m}$ be any tower of fields such that $\operatorname{dim}\left(q_{K_{i-1}}\right)_{\text {an }}>\operatorname{dim}\left(q_{K_{i}}\right)_{\text {an }}$ for each $1 \leq i \leq m$, then $m \leq h$.

It should be noted that the dimensions or types, respectively, of the forms $\left(q_{K_{j}}\right)_{\mathrm{an}}, 1 \leq j \leq m$ above might not necessarily be found among the dimensions or types, respectively, of the higher kernel forms $q_{i}, 1 \leq i \leq h$ (see also Remark 8.15). However, we do have

Proposition 4.6. Let $q$ be an anisotropic form over $F$ of type $\left(r_{0}, s_{0}\right)$, and let $\left(r_{i}, s_{i}\right)$ be the type of the $i$-th kernel form $q_{i}$ in a standard splitting tower $F=F_{0} \subset$ $F_{1} \subset \cdots \subset F_{h}$ of $q$. Let $K / F$ be any field extension and let $(r, s)$ be the type of $\left(q_{K}\right)_{\text {an. }}$. Then there exists $i \in\{0, \cdots, h\}$ such that $r=r_{i}$. 
Proof. If $s_{0}=0,1$, then this has been shown in [26. Th. 1.3]. If $r_{0} \leq 1$ or if $r=r_{0}$, the result is trivial.

Suppose that $r_{0} \geq 2, s_{0} \geq 1$ and let $i \in\{0, \cdots, h-1\}$ be such that $r<r_{i}$. To get the desired result, it suffices to show that $r \leq r_{i+1}$. Now $r_{i} \geq 1$ and we have $F_{j+1}=F_{j}\left(q_{j}\right)$ for $0 \leq j \leq i$. Define inductively $K_{0}=K$ and $K_{j+1}=K_{j}\left(q_{j}\right)$ for $0 \leq j \leq i$. Clearly, $F_{j} \subset K_{j}$ and the nondefective part of $\left(q_{j}\right)_{K_{j}}$ is of dimension $\geq 2 r_{j}+1 \geq 3$ for all $0 \leq j \leq i$, so that indeed all $K_{j}, 0 \leq j \leq i+1$ are well defined and can be identified with the free compositum $K \cdot F_{j}$.

Note that

$$
\begin{aligned}
i_{W}\left(\left(q_{j}\right)_{K_{j}}\right) & =i_{W}\left(q_{K_{j}}\right)-i_{W}\left(q_{F_{j}}\right) \\
& \geq i_{W}\left(q_{K}\right)-i_{W}\left(q_{F_{j}}\right) \geq\left(r_{0}-r\right)-\left(r_{0}-r_{j}\right)=r_{j}-r>0
\end{aligned}
$$

for $0 \leq j \leq i$. Therefore, $K_{j}\left(q_{j}\right) / K_{j}$ is purely transcendental for $0 \leq j \leq i$, hence $K_{i+1} / K$ is purely transcendental. Therefore, $r_{0}-r=i_{W}\left(q_{K}\right)=i_{W}\left(q_{K_{i+1}}\right) \geq$ $i_{W}\left(q_{F_{i+1}}\right)=r_{0}-r_{i+1}$, and thus $r \leq r_{i+1}$ as desired.

Suppose now that $q$ is nonsingular. Then the degree of $q$ is defined as follows. We put $\operatorname{deg}(q)=\infty$ if $q$ is hyperbolic. Otherwise, $h(q) \geq 1$ and $q_{h-1}$ becomes hyperbolic over its own function field, which implies that $q_{h-1}$ is similar to an $n$ fold Pfister form over $F_{h-1}$ for some $n \geq 0$. We then put $\operatorname{deg}(q)=n$. The set $J_{n} F=\left\{q \in W_{q} F \mid \operatorname{deg}(q) \geq n\right\}$ is a $W F$-submodule of $W_{q} F$ with $I^{n} W_{q} F \subset J_{n} F$. Aravire and Baeza have shown in [6], [7] that in fact $J_{n} F=I^{n} W_{q} F$. It should be noted that the corresponding result $I^{n} F=J_{n} F$ in characteristic $\neq 2$ is known to be true for $n \leq 5$ due to the work on the Milnor Conjecture in small degrees by Merkurjev, Suslin and Rost (see, e.g. [21, Th. 2.8, Remarque]), and for all $n$ due to Orlov, Vishik and Voevodsky [34] (see also [4, Theorem 1.5]).

Another important theorem by Aravire and Baeza which we will use is the following (cf. [7, Theorem 5.3]):

Theorem 4.7. Let $\left\langle\left\langle a_{1}, \cdots, a_{n}\right\rangle\right\rangle$ be an anisotropic $n$-fold quasi-Pfister form. Let $\varphi \in I^{n+1} W_{q} F$ and $K=F\left(\left\langle\left\langle a_{1}, \cdots, a_{n}\right\rangle\right\rangle\right)$. If $\varphi_{K} \in I^{n+2} W_{q} K$, then there exists $b \in F$ such that $\varphi \equiv\left\langle\left\langle a_{1}, \cdots a_{n}, b\right]\right] \bmod I^{n+2} W_{q} F$.

4.3. The Arf-invariant and the Clifford algebra. For basic facts on Clifford algebras and quaternion algebras which we state without further comment, we refer the reader to [1], 32], [14].

Let $q$ be a quadratic form defined on the vector space $V$ and let $T(V)=$ $\bigoplus_{i=0}^{\infty} V^{\otimes n}$ denote the tensor algebra of $V$. Let $J(q)$ be the ideal in $T(V)$ generated by elements of type $x \otimes x-q(x), x \in V$. Then the Clifford algebra $C(q)$ of $q$ is defined to be the quotient $T(V) / J(q)$. This algebra has a natural $\mathbb{Z} / 2 \mathbb{Z}$ gradation, and the subalgebra $C_{0}(q)$ of degree 0 elements is called the even Clifford algebra.

If $q$ is nonsingular, then $C(q)$ is a central simple algebra over $F$ which, up to isomorphism, only depends on the isometry class of $q$, and the center $Z(q)$ of $C_{0}(q)$ is a separable quadratic algebra over $F$; i.e., there exists $\delta \in F$ with $Z(q)=F[X] /\left(X^{2}+X+\delta\right)$. If we put $\wp(a)=a^{2}+a$, then $\wp(F)=\{\wp(a) \mid a \in F\}$ is an additive subgroup of $F$, and the class of $\delta$ modulo $\wp(F)$ is an invariant of the isometry class of $q$ and will be called the Arf-invariant of $q$, denoted by $\Delta(q)$. By abuse of notation, we will often identify $\Delta(q)$ with a representative in $F$ of its class in $F / \wp(F)$. 
More explicitly, if $q \cong a_{1}\left[1, b_{1}\right] \perp \cdots \perp a_{n}\left[1, b_{n}\right]$, then $\Delta(q)=b_{1}+\cdots+b_{n} \in$ $F / \wp(F)$, and $C(q)=\left[b_{1}, a_{1}\right)_{F} \otimes \cdots \otimes\left[b_{n}, a_{n}\right)_{F}$, where $[b, a)_{F}\left(a \in F^{*}, b \in F\right)$ denotes the quaternion algebra generated by two elements $u, v$ over $F$ subject to the relations $u^{2}=a, v^{2}+v=b, u v=(v+1) u$. This is a 4-dimensional central simple algebra which is trivial in the Brauer group iff $a$ is a norm for the extension $F[X] /\left(X^{2}+X+b\right)$ over $F$. For quaternion algebras, one has $[b, a)_{F} \otimes\left[b^{\prime}, a^{\prime}\right)_{F} \cong$ $\left[b, a a^{\prime}\right)_{F} \otimes\left[b+b^{\prime}, a^{\prime}\right)_{F}$. In particular, considered as a symbol in the Brauer group, it is additive in the first and multiplicative in the second slot. Two central simple algebras $A, B$ are said to be similar, $A \sim B$, if they represent the same element in the Brauer group $\operatorname{Br}(F)$.

We will need the following results.

Proposition 4.8 (Albert 2 Theorem 28, p. 108]). Let $A$ be a central simple algebra over $F$ and let $K=F\left(\sqrt{b_{1}}, \cdots, \sqrt{b_{n}}\right),[K: F]=2^{n}$. If $A_{K} \sim 0$, then there exist $a_{1}, \cdots, a_{n} \in F$ such that $A \sim\left[a_{1}, b_{1}\right)_{F} \otimes \cdots \otimes\left[a_{n}, b_{n}\right)_{F}$.

Theorem 4.9 (Sah [36, Theorem 2]). Let $q$ be a nonsingular quadratic form over $F$.

(i) $q \in I^{2} W_{q} F$ iff $\Delta(q)=0 \in F / \wp(F)$.

(ii) If $q \in I^{2} W_{q} F$, then $q \in I^{3} W_{q} F$ iff $C(q) \sim 0$.

\section{FitzGerald's TheOREM}

Let $q$ be a nonsingular nonhyperbolic form over $F$. We define

$$
N(q)=\operatorname{dim} q-2^{\operatorname{deg} q} .
$$

With this definition, we now give our characteristic 2 version of Fitzgerald's theorem [15, Th. 1.6].

Theorem 5.1. Let $q, \eta_{r}, \varphi_{r}$ be nonsingular forms over $F$, and let $\sigma$ be a totally singular form over $F$. Let $\rho$ be nonsingular with $\sigma \prec \rho$ and $\operatorname{dim} \rho=2 \operatorname{dim} \sigma$, i.e., $\rho$ is a nonsingular completion of $\sigma$. Suppose that

(i) $q, \eta=\eta_{r} \perp \sigma, \varphi=\varphi_{r} \perp \sigma$ are anisotropic,

(ii) $\operatorname{dim} q, \operatorname{dim} \varphi \geq 2$,

(iii) $q \sim \varphi_{r} \perp \eta_{r} \perp \rho$,

(iv) $\operatorname{dim} \eta<\operatorname{dim} \varphi+2^{\operatorname{deg} q}$.

Then $q$ is hyperbolic over $F(\varphi)$ if and only if $q$ is similar to a Pfister form and $q \cong \varphi_{r} \perp \eta_{r} \perp \rho$.

Proof. "if": Suppose that $q$ is similar to a Pfister form (which is anisotropic by hypothesis), and that $q \cong \varphi_{r} \perp \eta_{r} \perp \rho$. Since $\varphi \prec q$, $\operatorname{dim} \varphi \geq 2$, we have that $q$ becomes isotropic and hence hyperbolic over $F(\varphi)$.

"only if": We will prove this in a sequence of claims. So from now on, we suppose that $q$ is hyperbolic over $F(\varphi)$.

Claim 5.1.1. $q \cong \varphi_{r} \perp \eta_{r} \perp \rho$.

Proof. Since $\varphi_{r} \perp \varphi_{r} \sim 0$ and $\rho \perp \sigma \sim \sigma$ (see Lemma 3.7), we have that $q \perp \varphi \sim \eta$. Now $\operatorname{dim}(q \perp \varphi) \geq \operatorname{dim} \varphi+2^{\operatorname{deg} q}>\operatorname{dim} \eta$ by assumption (iv). Hence, $q \perp \varphi$ is isotropic and there exists an $a \in F^{*}$ represented both by $q$ and $\varphi$ because both these forms are anisotropic. Since $q_{F(\varphi)}$ is hyperbolic, it follows from the domination theorem that $\varphi \cong a^{2} \varphi \prec q$. 
Thus, there exist a nonsingular form $\tau$ and a nonsingular completion $\rho^{\prime}$ of $\sigma$ with $q \cong \varphi_{r} \perp \tau \perp \rho^{\prime} \sim \varphi_{r} \perp \eta_{r} \perp \rho$. Witt cancellation for nonsingular forms implies $\tau \perp \rho^{\prime} \sim \eta_{r} \perp \rho$. Now $\tau \perp \rho^{\prime}$ is anisotropic and $\tau \perp \sigma \prec \tau \perp \rho^{\prime}$. In particular, $\tau \perp \sigma$ is anisotropic. Since $\rho^{\prime} \perp \sigma \sim \rho \perp \sigma \sim \sigma$, we get $\tau \perp \sigma \sim \eta_{r} \perp \sigma$. Since the forms on both sides are anisotropic, we therefore obtain $\tau \perp \sigma \cong \eta_{r} \perp \sigma$ and thus $\operatorname{dim} \tau=\operatorname{dim} \eta_{r}$. Thus, the anisotropic form $q \cong \varphi_{r} \perp \tau \perp \rho^{\prime}$ and the form $\varphi_{r} \perp \eta_{r} \perp \rho$ have the same dimension, and since they are Witt-equivalent, it follows that $q \cong \varphi_{r} \perp \eta_{r} \perp \rho$.

After scaling, we may therefore assume that $1 \in D_{F}(q) \cap D_{F}(\varphi)$.

Since an anisotropic nonsingular form becomes hyperbolic over its own function field iff the form is similar to a Pfister form (Theorem 4.2), we get that $q$ is similar to a Pfister form (and thus isometric to a Pfister form as $1 \in D_{F}(q)$ ) iff $N(q)=0$. Thus, it suffices to show that under the above assumptions we cannot have $N(q)>$ 0 .

Suppose that $N(q)=m>0$. We will show by induction on $m$ that this will yield a contradiction.

Claim 5.1.2. Let $F_{1}=F(q)$ and $q_{1}=\left(q_{F_{1}}\right)_{\text {an. }}$. Then $\varphi_{F_{1}}$ is anisotropic, $q_{1}$ is a Pfister form of dimension $2^{\operatorname{deg} q}$, and $\varphi_{F_{1}} \prec q_{1}$.

Proof. $N(q)>0$ implies that $h(q) \geq 2$. Then $q_{1} \not 0$. Furthermore, $\sigma_{F_{1}}$ stays anisotropic (cf. [28, Cor.3.1]). In particular, $\varphi_{F_{1}}$ will stay nondefective, so that as a polynomial over $F_{1}, \varphi_{F_{1}}$ will be irreducible. It follows that the compositum of $F_{1}$ and $F(\varphi)$ is just $F_{1}(\varphi)$. Therefore, $\left(q_{1}\right)_{F_{1}(\varphi)}$ is hyperbolic. Since the function field of a nondefective isotropic form is purely transcendental over the ground field, and since the nonhyperbolic form $q_{1}$ becomes hyperbolic over $F_{1}(\varphi)$, we have that $\varphi_{F_{1}}$ is anisotropic. Clearly, $\operatorname{deg} q=\operatorname{deg} q_{1}$ and thus $N\left(q_{1}\right)<N(q)$.

Put $\eta_{1}=\left(\eta_{F_{1}}\right)_{\text {an }}$. As remarked above, the quasi-linear part $\sigma$ of $\eta$ will stay anisotropic (if $\operatorname{dim} \sigma>0$ ). Hence, there exists a nonsingular form $\eta_{1, r}$ over $F_{1}$ such that $\eta_{1} \cong \eta_{1, r} \perp \sigma$. Now over $F_{1}$ we have

$$
\varphi_{r} \perp \eta_{r} \perp \sigma \sim_{F_{1}} \varphi_{r} \perp \eta_{1, r} \perp \sigma .
$$

By Lemma 3.9 there exists a nonsingular completion $\rho_{1}$ of $\sigma$ over $F_{1}$ such that

$$
q_{1} \sim_{F_{1}} \varphi_{r} \perp \eta_{r} \perp \rho \sim_{F_{1}} \varphi_{r} \perp \eta_{1, r} \perp \rho_{1} .
$$

Replacing $q$ by $q_{1}, \eta$ by $\eta_{1}, \eta_{r}$ by $\eta_{1, r}, \varphi$ by $\varphi_{1}=\varphi_{F_{1}}, \varphi_{r}$ by $\varphi_{1, r}=\left(\varphi_{r}\right)_{F_{1}}, \sigma$ by $\sigma_{1}=\sigma_{F_{1}}, \rho$ by $\rho_{1}$, it is easily verified that these forms satisfy the hypotheses (i)-(iv). The same reasoning as above shows then that $q_{1} \cong \varphi_{1, r} \perp \eta_{1, r} \perp \rho_{1}$. Furthermore, since $N\left(q_{1}\right)<N(q)$, it follows by induction that $q_{1}$ is similar to a Pfister form, and hence isometric to a Pfister form since $1 \in D_{K}\left(\varphi_{1}\right)$ and $\varphi_{1} \prec q_{1}$. In particular, $\operatorname{dim} q_{1}=2^{\operatorname{deg} q_{1}}=2^{\operatorname{deg} q}$.

Claim 5.1.3. $D_{F}(q)=G_{F}(q)$.

Proof. Since $1 \in D_{F}(q)$ and $q$ anisotropic, we have $G_{F}(q) \subset D_{F}(q)=D_{F}^{*}(q)$. Suppose there exists an $x \in D_{F}(q) \backslash G_{F}(q)$. Let $\beta=\left(\langle\langle x\rangle\rangle_{b} \otimes q\right)_{\text {an }}$. By assumption, $\left\langle\langle x\rangle_{b} \otimes q\right.$ is isotropic but not hyperbolic. It follows readily that $D_{F}(q) \cap D_{F}(\beta) \neq \emptyset$. Also, $\operatorname{deg} \beta \geq 1+\operatorname{deg} q$ (see, e.g., [37, Ch.4, Theorem 7.5] in the case of characteristic $\neq 2$; the proof for the case of characteristic 2 is more or less identical).

Suppose that $\beta_{F_{1}} \sim 0$. The domination theorem and the fact that $D_{F}(q) \cap$ $D_{F}(\beta) \neq \emptyset$ imply that $q \subset \beta$. Then there exists a nonsingular form $q^{\prime}$ with 
$\beta \cong q \perp q^{\prime} \sim q \perp x q$. Now $\operatorname{dim} \beta<2 \operatorname{dim} q$, hence $\operatorname{dim} q^{\prime}<\operatorname{dim} q$. But we have $q^{\prime} \sim x q$, hence $q$ is isotropic, a contradiction. It follows that $\beta_{F_{1}} \nsim 0$. In particular, with $\beta_{1}=\left(\beta_{F_{1}}\right)_{\text {an }}$, we get $\operatorname{dim} \beta_{1} \geq 2^{\operatorname{deg} \beta} \geq 2^{1+\operatorname{deg} q}$.

Since $x \in D_{F}(q)$, we have $1 \in D_{F}(x q)$. Now $1 \in D_{F}(\varphi)$, and $x q$ becomes hyperbolic over $F_{1}=F(\varphi)$. The domination theorem implies that there exists a nonsingular form $\eta_{r}^{\prime}$ and a nonsingular completion $\rho^{\prime}$ of $\sigma$ such that $x q \cong \varphi_{r} \perp$ $\eta_{r}^{\prime} \perp \rho^{\prime}$ and $\operatorname{dim} \eta_{r}=\operatorname{dim} \eta_{r}^{\prime}$.

Over $F_{1}$ we have $\beta_{1} \sim q_{1} \perp x q_{1}$. Now $\varphi_{F_{1}} \prec q_{1}$ and, by the same reasoning as in the previous claim, $\varphi_{F_{1}} \prec x q_{1}$. In particular,

$$
2^{1+\operatorname{deg} q} \leq \operatorname{dim} \beta_{1}=\operatorname{dim}\left(q_{1} \perp x q_{1}\right) \text { an } \leq 2 \operatorname{dim} q_{1}-2 \operatorname{dim} \varphi<2^{1+\operatorname{deg} q},
$$

which is absurd. Hence, $D_{F}(q)=G_{F}(q)$.

Now let $K=F\left(X_{1}, \cdots, X_{n}\right)$ be the rational function field in $n=\operatorname{dim} q$ variables over $F$. Clearly, $\operatorname{deg} q_{K}=\operatorname{deg} q$, and working over $K$ instead of $F$, it is obvious that the forms $q_{K}, \varphi_{K},\left(\varphi_{r}\right)_{K}, \eta_{K},\left(\eta_{r}\right)_{K}, \sigma_{K}$ and $\rho_{K}$ satisfy the hypotheses (i)(iv). By the previous claim, we therefore have $D_{K}(q)=G_{K}(q)$. Hence $q_{K} \cong$ $q\left(X_{1}, \cdots, X_{n}\right) q_{K}$, i.e., the anisotropic form $q$ is strictly multiplicative. It follows that $q$ is a Pfister form, hence $N(q)=0$, a contradiction to the assumption $N(q)>0$ and the proof of the theorem is completed.

Remark 5.2. To get Fitzgerald's original version in characteristic $\neq 2$ from our version above, one simply has to omit $\sigma$; i.e., $\varphi$ and $\eta$ are nonsingular, and one has to replace "+" by "-" in certain obvious places in the proof. Furthermore, in the original version, the condition on $q$ being anisotropic was replaced by $q$ nonhyperbolic and $q \cong \varphi \perp \eta$, and condition (iv) by the then equivalent condition $N(q)<2 \operatorname{dim} \varphi$. As a consequence, the hyperbolicity of $q$ over $F(\varphi)$ becomes equivalent to $q$ being similar to an anisotropic Pfister form.

Our proof follows along the same lines as Fitzgerald's original proof. Certain modifications became necessary because of the possibility of having singular forms and dominated forms. On the other hand, some of the original arguments could be simplified by using the domination theorem more prominently in certain places.

The following corollary is a characteristic 2 version of a result due to Knebusch [27, Cor. 13.4].

Corollary 5.3. Let $\varphi$ and $q$ be forms such that $q$ is nonsingular and anisotropic, $\varphi$ is nondefective of type $(r, s)$, and $3 \operatorname{dim} \varphi+s>\operatorname{dim} q$. If $q_{F(\varphi)}$ is hyperbolic, then $q$ is similar to a Pfister form.

Proof. Let $h$ be the height of $q$ and $K=F_{h-1}$ its leading field. If $h \geq 2$, then $K$ is obtained by successively taking function fields of nonsingular forms. By Lemma 4.1. $\varphi$ stays nondefective over $K$ (which trivially also holds if $h=1$, i.e., $K=F$ ). Let $d=\operatorname{deg}(q)$ and let $\tau \in G P_{d} K$ be the leading form of $q$. Now $q_{F(\varphi)} \sim 0$, hence $q_{K(\varphi)} \sim \tau_{K(\varphi)} \sim 0$ and by the domination theorem 4.2(i), there exists $a \in K^{*}$ such that $a \varphi_{K} \prec \tau$. Now $\tau$ is nonsingular and $\varphi_{K}$ is nondefective of type $(r, s)$, hence $\operatorname{dim} \varphi+s=2 r+2 s \leq 2^{d}=\operatorname{dim} \tau$ by Lemma 3.1

Now $q_{F(\varphi)} \sim 0$ implies, again by the domination theorem, $b \varphi \prec q$ for some $b \in F^{*}$, and after scaling, we may assume $b=1$. By Lemma [3.1, we can write $\varphi \cong \varphi_{r} \perp \sigma$ and $q \cong \varphi_{r} \perp \rho \perp \eta_{r}$ with $\varphi_{r}, \eta_{r}$ nonsingular, $\sigma$ totally singular, and $\rho$ 
an n.s.c. of $\sigma$. By putting $\eta \cong \eta_{r} \perp \sigma$, we note that $\varphi$ and $\eta$ are anisotropic, and furthermore,

$$
\operatorname{dim} \eta=\operatorname{dim} q-\operatorname{dim} \varphi<3 \operatorname{dim} \varphi+s-\operatorname{dim} \varphi=\operatorname{dim} \varphi+2 r+2 s \leq \operatorname{dim} \varphi+2^{d} .
$$

By Theorem [5.1, it follows that $q$ is similar to an anisotropic Pfister form.

Remark 5.4. At least in the case $s=0$, the above restriction $\operatorname{dim} q<3 \operatorname{dim} \varphi+s=$ $3 \operatorname{dim} \varphi$ cannot be weakened in general to obtain the same conclusion. In fact, let $\varphi \in P_{n} F, n \geq 1$, and suppose that $q \cong \varphi \perp a \varphi \perp b \varphi$ is anisotropic for some $a, b \in F^{*}$. One has $\operatorname{dim} q=3 \operatorname{dim} \varphi$ and clearly $q_{F(\varphi)} \sim 0$, but $q$ is not similar to a Pfister form for dimension reasons (see also [27, Example, p. 42]).

\section{Pfister neighbors and an analogue of a theorem BY KNEBUSCH IN CHARACTERISIC 2}

A form $q$ is said to be a Pfister neighbor if there exist $\pi \in P_{n} F$ for some $n$ and an $a \in F^{*}$ such that $q \prec a \pi$ and $\operatorname{dim} q>2^{n-1}$. We then say that $q$ is a Pfister neighbor of $\pi$. In this case, the Pfister form $\pi$ is uniquely determined by $q$ up to isometry, and $q$ is isotropic iff $\pi$ is isotropic (and hence hyperbolic); cf. 28, Prop. 3.1]. Furthermore, it is rather obvious that if $q$ is a Pfister neighbor of type $(r, s)$, then there exists an integer $n \geq 1$ such that $2 r+s>2^{n-1}$ and $2 r+2 s \leq 2^{n}$. In particular, $q$ cannot be totally singular. If $q$ is a Pfister neighbor of $\pi \in P_{n} F$, and if $x, y \in F^{*}$ such that $q \prec x \pi$ and $q \prec y \pi$, then the roundness of $\pi$ implies that $x \pi \cong y \pi$. This together with the uniqueness of $\pi$ implies that $q_{x \pi}^{c}$ only depends on the Pfister neighbor $q$ (see also [29] Prop. 5.2]). By abuse of language and notation, we will call it the complement of the Pfister neighbor $q$ and write $q^{c}$ for short.

Pfister forms can be characterized up to similarity by the property that they become hyperbolic over their own function field; see Theorem 4.2 (ii). The behaviour of Pfister neighbors over their own function field is equally well understood.

Proposition 6.1. Let $q$ be an anisotropic Pfister neighbor. Then $\left(q_{F(q)}\right)_{\text {an }} \cong q_{F(q)}^{c}$. In particular, the anisotropic part of $q$ over $F(q)$ is defined over $F$.

Proof. Let $n$ and $\pi \in G P_{n} F$ such that over $F, q \prec \pi$ and $\operatorname{dim} q>2^{n-1}$. $\pi$ is anisotropic as $q$ is anisotropic, hence $q^{c}$ is anisotropic as well. Furthermore, $\pi \perp q \sim q^{c}$. Now $\pi_{F(q)}$ is isotropic because $q_{F(q)}$ is isotropic, hence $\pi_{F(q)}$ is hyperbolic, and we get $\left(q_{F(q)}\right)$ an $\sim q_{F(q)}^{c}$. But $\operatorname{dim} q>2^{n-1}>2^{n}-\operatorname{dim} q=\operatorname{dim} q^{c}$, and by Theorem 4.4, the anisotropic form $q^{c}$ stays anisotropic over $F(q)$. Thus, $\left(q_{F(q)}\right)_{\mathrm{an}} \cong q_{F(q)}^{c}$.

In characteristic $\neq 2$, Knebusch [24 Theorem 7.13] has shown that the converse of the last statement in the previous proposition also holds, more precisely, an anisotropic form $q$ with the property that its anisotropic part over $F(q)$ is defined over $F$ must be a Pfister neighbor, so that together with the above result, anisotropic Pfister neighbors can be characterized by this property. This also holds in characteristic 2 for nonsingular forms as follows readily from Theorem 5.1 (see also Theorem 6.6] below for more details). However, for singular forms the converse fails in general.

Example 6.2. Let $\sigma$ be a totally singular anisotropic form. Then $\left(\sigma_{F(\sigma)}\right)_{\text {an }}$ is defined over $F$. This is clear by Lemma [2.2. However, $\sigma$ is not a Pfister neighbor as we already mentioned above. 
Example 6.3. Here, we construct counterexamples of forms of type $(1, s)$.

Let $q \cong q_{r} \perp \sigma$ be an anisotropic form of type $(1, s)$ with $q_{r}$ nonsingular and $\sigma$ totally singular. Lemma 4.1 immediately implies that $\left(q_{F(q)}\right)_{\text {an }} \cong \sigma_{F(q)}$. Now if $q$ is a Pfister neighbor, then Proposition 6.1 implies that $q^{c}=\sigma$, hence there exists an $n$ with $\operatorname{dim} q=2+s>2^{n-1}$ and $2+2 s=\operatorname{dim} q+\operatorname{dim} q^{c}=2^{n}$. This implies $s=2^{n-1}-1$. Thus, any anisotropic form of type $(1, s)$ with $s+1$ not a 2 -power will be a non-Pfister neighbor with the property that its anisotropic part over $F(q)$ is defined over $F$.

Examples of this type have first been noted by Knebusch 25$]$ and, independently, the second author [29, Prop. 5.12].

Now nondefective forms of type $(1,1)$ are easily seen to be Pfister neighbors. However, there are fields such that for any $n \geq 3$ there exist anisotropic forms of type $\left(1,2^{n-1}-1\right)$ which are not Pfister neighbors. For one can construct fields with anisotropic nonsingular forms of arbitrarily large dimension, i.e. $u(F)=\infty$, but for which $I^{3} W_{q} F=0$. This can be done as in the characteristic $\neq 2$ case (where it was first done by Merkurjev [33]) using the methods in [32]. Over such a field, any anisotropic nonsingular form of dimension $2^{n}, n \geq 3$, clearly contains a dominated form of type $\left(1,2^{n-1}-1\right)$. This anisotropic form of dimension $2^{n-1}+1$ cannot be a Pfister neighbor as $n$-fold Pfister forms are hyperbolic for $n \geq 3$ because $I^{3} W_{q} F=0$.

Example 6.4. The construction we now describe can be used to produce counterexamples of type $(r, s)$ subject to the following conditions: There exist integers $n, m, t \geq 0$ with $n<m, 2 r+t>2^{n}, 2 r+2 t \leq 2^{n+1}$, and $s=t+2^{m}$. In particular, we get counterexamples of type $\left(2^{n}, 2^{n+1}\right)$.

Consider a Pfister form $\pi=\left\langle\left\langle a_{1}, \cdots, a_{n} ; b\right]\right]$ and an anisotropic totally singular Pfister form $\tau=\left\langle\left\langle a_{1}, \cdots, a_{m}\right\rangle\right\rangle$ with $m>n$. Let $x \in F^{*}$ and put $\varphi=\pi \perp x \tau$. Let $E / F$ be any field extension such that $\tau_{E}$ stays anisotropic but such that $\varphi_{E}=$ $\pi_{E} \perp x \tau_{E}$ is isotropic. Then there exists $y \in D_{E}(\pi) \cap D_{E}(x \tau)$. The roundness of $\pi$ and $\tau$ implies that $\varphi_{E} \cong y\left(\pi_{E} \perp \tau_{E}\right)$.

Now $\pi$ is an orthogonal sum of the forms $\left(\prod_{i \in I} a_{i}\right)[1, b]$ with $I \subset\{1, \cdots, n\}$, and $\tau$ is an orthogonal sum of the forms $\left\langle\prod_{j \in J} a_{j}\right\rangle$ with $J \subset\{1, \cdots, m\}$. Since $[u, v] \perp\langle u\rangle \cong \mathbb{H} \perp\langle u\rangle$, it follows readily that $\pi \perp \tau \sim \tau$, hence $\varphi_{E} \sim y \tau_{E} \cong x \tau_{E}$.

Now let $\psi \prec \pi$ be a Pfister neighbor of $\pi$ with complement $\psi^{c}$ and let $\eta=\psi \perp x \tau$ for some $x \in F^{*}$. Thus, if $E$ is a field extension with $\tau_{E}$ anisotropic, and if $\psi_{E} \perp x \tau_{E}$ is isotropic, then the above shows that $\eta_{E} \sim \psi_{E}^{c} \perp x \tau_{E}$ because $\pi \perp \psi^{c} \sim \psi$.

Suppose now that $\pi$ is anisotropic and that $x \in F^{*}$ is such that $\pi \perp x \tau$ is anisotropic as well. In particular, this implies that $\eta$ and $\psi^{c} \perp x \tau$ are anisotropic. Note that $\eta$ is not totally singular as the Pfister neighbor $\psi$ is not totally singular. Also, $\eta$ will not be a Pfister neighbor. Indeed, suppose $\eta$ is a Pfister neighbor. Now $2^{m+1} \geq 2^{m}+2^{n+1} \geq \operatorname{dim} \eta>2^{m}+2^{n}$; thus, $\eta$ is necessarily a Pfister neighbor of an $(m+1)$-fold Pfister form. If $(r, s)$ is the type of $\eta$, this implies that $r+2 s \leq 2^{m+1}$, a contradiction as $r \geq 1$ and $s \geq 2^{m}$.

Let $E=F(\eta)$. Then, by the above, $\eta_{E} \sim\left(\psi^{c} \perp x \tau\right)_{E}$. Thus, if $\left(\psi^{c} \perp x \tau\right)_{E}$ is anisotropic, this gives an example of an anisotropic non-Pfister neighbor $\eta$ whose anisotropic part over $F(\eta)$ is defined over the base field $F$.

This certainly holds if $\psi^{c} \perp x \tau$ is totally singular (cf. Lemma 4.1), i.e., $\psi^{c}$ is totally singular. This is the case if and only if the Pfister neighbor $\psi$ is of type $\left(r^{\prime}, s^{\prime}\right)$ with $2 r^{\prime}+2 s^{\prime}=2^{n+1}$. (Knebusch [25] calls such Pfister neighbors close 
Pfister neighbors.) This incidentally yields examples of non-Pfister neighbors of nondefective height 1 .

To construct explicit situations with $\left(\psi^{c} \perp x \tau\right)_{F(\eta)}$ anisotropic, let $\pi$ and $\tau$ be as above, anisotropic, and with $a_{i}, b \in F_{0}$, and let $\psi \prec \pi$ be a Pfister neighbor over $F_{0}$. Let $F=F_{0}(x)$ with $x$ an indeterminate. By the usual degree argument, we see that $\pi \perp x \tau$ is anisotropic. Let $K=F_{0}(\psi)$. Then $\psi_{K}^{c}$ is anisotropic (cf. Proposition 6.1), and so is the totally singular form $\tau_{K}$. Hence, over $F(\psi)=F_{0}(\psi)(x)$, we have that $\left(\psi^{c} \perp x \tau\right)_{F(\psi)}$ is anisotropic. Now $\eta_{F(\psi)}$ is nondefective and isotropic, hence $F(\psi)(\eta) / F(\psi)$ is purely transcendental; thus $\left(\psi^{c} \perp x \tau\right)_{F(\psi)(\eta)}$ is anisotropic and therefore, $\left(\psi^{c} \perp x \tau\right)_{F(\eta)}$ is anisotropic as desired.

A natural question is to ask under which conditions the converse of the last statement in Proposition 6.1 holds. In all the examples above, the counterexamples are of type $(r, s)$ with $s \geq 2 r$. In view of this, we venture the following

Conjecture 6.5. Let $q$ be an anisotropic form such that the anisotropic part of $q$ over $F(q)$ is defined over $F$. If $q$ is of type $(r, s)$ with $s<2 r$, then $q$ is a Pfister neighbor.

Theorem 6.6. Conjecture 6.5 is true if $s \leq 4$ or if $s=5$ and there exist $a, b, c \in F$ such that the quasi-linear part of $q$ is similar to $\langle\langle a, b\rangle\rangle \perp\langle c\rangle$.

To prove the theorem, we need some lemmas.

Lemma 6.7. Let $\varphi \cong \varphi_{r} \perp \sigma$ be anisotropic of type $(r, s), r \geq 1$, with $\sigma=\mathrm{ql}(\varphi)$. Suppose that the anisotropic part of $\varphi_{F(\varphi)}$ is defined over $F$. Then there exists a form $\eta \cong \eta_{r} \perp \sigma$ over $F$ with $\eta_{r}$ nonsingular such that

- $\left(\varphi_{F(\varphi)}\right)_{\text {an }} \cong \eta_{F(\varphi)}$, and

- $\left(\varphi_{r} \perp \eta_{r} \perp \sigma\right)_{F(\varphi)} \sim \sigma_{F(\varphi)}$.

If, furthermore, there exists a nonsingular completion $\rho$ of $\sigma$ over $F$ such that $q \cong \varphi_{r} \perp \eta_{r} \perp \rho \in I^{n} W_{q} F$ with $2^{n}>2 s$, then $q$ is anisotropic and similar to a Pfister form. In particular, $\varphi$ is a Pfister neighbor.

Proof. Let $K=F(\varphi)$. By Lemma $4.1 \sigma_{K} \cong \mathrm{ql}\left(q_{K}\right)$. Hence, we may assume that the anisotropic part over $K$ is defined over $F$ by a form $\eta$ whose quasi-linear part is $\sigma$, i.e., $\eta \cong \eta_{r} \perp \sigma$ with $\eta_{r}$ nonsingular.

Now $\left(\varphi_{r} \perp \sigma\right)_{K} \sim\left(\eta_{r} \perp \sigma\right)_{K}$, hence $\left(\varphi_{r} \perp \eta_{r} \perp \sigma\right)_{K} \sim \sigma_{K}$.

Let $\rho$ be an n.s.c. of $\sigma$ with $q \cong \varphi_{r} \perp \eta_{r} \perp \rho \in I^{n} W_{q} F$ and $2^{n}>2 s$. By Lemma 3.9, there exists over $K$ an n.s.c. $\tau$ of $\sigma_{K}$ such that $q_{K} \sim \tau_{K} \in I^{n} W_{q} F$. Now $\operatorname{dim} \tau=2 s<2^{n}$, and the Arason-Pfister Hauptsatz implies $q_{K} \sim \tau \sim 0$. It follows now readily from Theorem 5.1 that $q$ is anisotropic and similar to a Pfister form. Since $\operatorname{dim} \varphi>\operatorname{dim} \eta$ and $\operatorname{dim} \varphi+\operatorname{dim} \eta=\operatorname{dim} q$, we have $\varphi \prec q$ and $2 \operatorname{dim} \varphi>\operatorname{dim} q$. Hence, $\varphi$ is a Pfister neighbor.

Lemma 6.8. Let $\varphi \cong \varphi_{r} \perp\left\langle 1, a_{2}, \cdots, a_{s}\right\rangle$ be nondefective of type $(r, s)$ with $s \geq$ 2 and $\varphi_{r}$ nonsingular. Suppose that $2 \leq t \leq s$ is such that, after reindexing, $\left[F\left(\sqrt{a_{2}}, \cdots, \sqrt{a_{s}}\right): F\right]=2^{t-1}$ and $F\left(\sqrt{a_{2}}, \cdots, \sqrt{a_{s}}\right)=F\left(\sqrt{a_{2}}, \cdots, \sqrt{a_{t}}\right)$. Let $q$ be nondefective of type $\left(r^{\prime}, s^{\prime}\right)$ with $\operatorname{dim} q \geq 5$ and $r^{\prime} \geq 2$. If $\left(\varphi_{F(q)}\right)$ an is totally singular, then there exist $b_{1}, \cdots, b_{t} \in F^{*}$ such that $\psi=\varphi_{r} \perp\left[1, b_{1}\right] \perp a_{2}\left[1, b_{2}\right] \perp$ $\cdots \perp a_{t}\left[1, b_{t}\right] \perp(t-s) \times \mathbb{H}$ is an n.s.c. of $\varphi$ with $\psi \in I^{3} W_{q} F$. 
Proof. Let $K=F\left(\sqrt{a_{2}}, \cdots, \sqrt{a_{t}}\right)$. By assumption, $\left\langle 1, a_{2}, \cdots, a_{s}\right\rangle_{K} \sim\langle 1\rangle_{K}$.

Let $q^{\prime}$ be the nondefective part of $q$ over $K$. Then $q^{\prime}$ is of type $\left(r^{\prime}, s^{\prime \prime}\right)$ and $\operatorname{dim} q^{\prime} \geq 5$. Indeed, if $r^{\prime} \geq 3$, this is clear, and if $r^{\prime}=2$, then $s^{\prime} \geq 1$ and hence $s^{\prime} \geq s^{\prime \prime} \geq 1$. Hence, $K(q) / K\left(q^{\prime}\right)$ is purely transcendental of transcendence degree $s^{\prime}-s^{\prime \prime}$.

By assumption, $\varphi_{F(q)} \sim\left\langle 1, a_{2}, \cdots, a_{s}\right\rangle_{F(q)}$, thus $\varphi_{K(q)} \sim\left\langle 1, a_{2}, \cdots, a_{s}\right\rangle_{K(q)} \sim$ $\langle 1\rangle_{K(q)}$, and since anisotropic forms stay anisotropic over purely transcendental extensions, we obtain $\left(\varphi_{r} \perp\langle 1\rangle\right)_{K\left(q^{\prime}\right)} \sim \varphi_{K\left(q^{\prime}\right)} \sim\langle 1\rangle_{K\left(q^{\prime}\right)}$. Let $\Delta=\Delta\left(\varphi_{r}\right)$. By Lemma 3.9 there exists $a \in K\left(q^{\prime}\right)$ such that $\left(\varphi_{r} \perp[1, \Delta]\right)_{K\left(q^{\prime}\right)} \sim[1, a]$. Now $\varphi_{r} \perp[1, \Delta] \sim[1, a] \in I^{2} W_{q} K\left(q^{\prime}\right)$ by Theorem 4.9 and therefore, by the ArasonPfister Hauptsatz, $[1, a] \sim 0$. In particular, $C\left(\varphi_{r} \perp[1, \Delta]\right)$ splits over $K\left(q^{\prime}\right)$.

Since $K\left(q^{\prime}\right) / K$ can be obtained as a purely transcendental extension followed by a separable quadratic extension, the index of $C\left(\varphi_{r} \perp[1, \Delta]\right)_{K}$ can only be 1 or 2. If it is 2 , then the class of $C\left(\varphi_{r} \perp[1, \Delta]\right)$ in $\operatorname{Br}(K)$ is given by a quaternion division algebra which stays division over $K\left(q^{\prime}\right)$ as $q_{K}^{\prime}$ is nondefective and $\operatorname{dim} q^{\prime} \geq 5$ (cf. [32, Theorem 3]), a contradiction. Hence, $C\left(\varphi_{r} \perp[1, \Delta]\right)_{K}$ is split, and by Albert's result (Proposition 4.8), it follows that there exist $b_{2}, \cdots, b_{t} \in F$ with $C\left(\varphi_{r} \perp[1, \Delta]\right) \sim\left[b_{2}, a_{2}\right)_{F} \otimes \cdots \otimes\left[b_{t}, a_{t}\right)_{F}$. Let $b_{1}=\Delta+b_{2}+\cdots+b_{t}$ and put

$$
\psi=\varphi_{r} \perp\left[1, b_{1}\right] \perp a_{2}\left[1, b_{2}\right] \perp \cdots \perp a_{t}\left[1, b_{t}\right] \perp(s-t) \times \mathbb{H} .
$$

Then one verifies rapidly that $\psi \in I^{3} W_{q} F$ and that $\psi$ is a nonsingular completion of $\varphi$.

Proof of Theorem [6.6] Let $\varphi \cong \varphi_{r} \perp \sigma$ be anisotropic of type $(r, s)$ with quasilinear part $\sigma$ and $2 r>s$. Suppose that $\left(\varphi_{F(\varphi)}\right)_{\text {an }} \cong \eta$ for some $F$-form $\eta$. Then, by Lemma 6.7 we may assume that $\eta \cong \eta_{r} \perp \sigma$ with $\eta_{r}$ nonsingular, $\operatorname{dim} \eta_{r}<\operatorname{dim} \varphi_{r}$.

The case $s=0$. In this case, $2>2 s=0$. Since $\varphi \perp \eta \cong \varphi_{r} \perp \eta_{r} \in I W_{q} F$, it follows from Lemma 6.7 that $\varphi \perp \eta$ is similar to an anisotropic Pfister form of which $\varphi$ is a Pfister neighbor.

The case $s=1$. After scaling, we may assume that $\sigma \cong\langle 1\rangle$. Let $d=\Delta\left(\varphi_{r}\right)+$ $\Delta\left(\eta_{r}\right)$ Then $\rho \cong[1, d]$ is a regular completion of $\sigma$, and by putting $q:=\varphi_{r} \perp \eta_{r} \perp \rho$, it follows from Theorem 4.9 that $q \in I^{2} W_{q} F$. Now $2^{2}>2 s=2$, hence Lemma 6.7 implies that $q$ is a Pfister form (note that $q$ represents 1 ) and that $\varphi$ is a Pfister neighbor of $q$.

The case $2 \leq s \leq 3.2 r>s$ implies that $r \geq 2$ and $\operatorname{dim} \varphi \geq 6$. Let $K=F(\varphi)$. By assumption $\left(\varphi_{r} \perp \eta_{r} \perp \sigma\right)_{K} \sim \sigma_{K}$. By Lemma 6.8 there exists an n.s.c. $\rho$ of $\sigma$ such that for $q=\varphi_{r} \perp \psi_{r} \perp \rho$ we get $q \in I^{3} W_{q} F$. Since $2^{3}>6 \geq 2 s$, Lemma 6.7 yields that $q$ is similar to an anisotropic Pfister form of which $\varphi \cong \varphi_{r} \perp \sigma$ is a Pfister neighbor.

The case $4 \leq s \leq 5$. By assumption in the case $s=5$ and after scaling, we may assume that $\sigma \cong\langle 1, a, b, c\rangle$ or $\sigma \cong\langle 1, a, b, a b, c\rangle, a, b, c \in F^{*}$.

Let $L=F(\langle 1, a, b, a b\rangle)$. By Theorem 8.11(ii), $\langle 1, a, b, a b\rangle_{L} \sim\langle 1, a\rangle_{L}$. Let $\sigma^{\prime}=$ $\left(\sigma_{L}\right)_{\text {an }}$. The above shows that $\sigma^{\prime} \cong\langle 1, a\rangle$ or $\sigma^{\prime} \cong\langle 1, a, c\rangle$.

By Lemma 6.8 there exists an n.s.c. $\rho$ of $\sigma$ such that $\psi:=\varphi_{r} \perp \eta_{r} \perp \rho \in I^{3} W_{q} F$.

Now put $M=L(\varphi)=K(\langle 1, a, b, a b\rangle)$. Since $\left(\varphi_{r} \perp \eta_{r} \perp \sigma\right)_{K} \sim \sigma_{K}$, there exists an n.s.c. $\rho^{\prime}$ of $\sigma$ over $M$ such that $\psi_{M} \sim \rho^{\prime}$. We have $i_{\mathrm{ti}}\left(\sigma_{M}\right) \geq s-3$, and $\sigma_{M} \prec \rho^{\prime}$ with $\rho^{\prime}$ nonsingular. Hence, $i_{W}\left(\rho^{\prime}\right) \geq s-3$ and $\operatorname{dim} \rho_{\text {an }}^{\prime} \leq 2 s-2(s-3)=6$. The Arason-Pfister Hauptsatz implies that $\rho^{\prime} \sim 0$. 
Clearly, $\operatorname{deg} \psi_{L} \geq 3$ as $\psi_{L} \in I^{3} W_{q} L=J_{3} L$. We claim that $\psi_{L} \in I^{4} W_{q} L=J_{4} L$. Suppose that $\operatorname{deg} \psi_{L}=3$. Let $N$ be the leading field of $\psi_{L}$ and let $\pi \in G P_{3} N$ be the leading form. We have $\psi_{N} \sim \pi$ and $0 \sim \psi_{N(\varphi)} \sim \pi_{N(\varphi)}$. Let $\varphi^{\prime}$ be the nondefective part of $\varphi_{L}$ which, by the above, is of type $\left(r, s^{\prime}\right)$ with $s^{\prime}=\operatorname{dim} \sigma^{\prime} \in\{2,3\}$ and $2 r=\operatorname{dim} \varphi_{r} \geq 6$. Since $N(\varphi) / N\left(\varphi^{\prime}\right)$ is purely transcendental, we also have $0 \sim$ $\psi_{N\left(\varphi^{\prime}\right)} \sim \pi_{N\left(\varphi^{\prime}\right)}$. Now $N$ is obtained from $L$ by taking function fields of nonsingular forms, hence the nondefective form $\varphi^{\prime}$ stays nondefective over $N$ (Lemma 4.1). It follows from the domination theorem (Theorem 4.2) that there exists $x \in N^{*}$ with $x \varphi^{\prime} \prec \pi$. In particular, $10 \leq 2 r+2 s^{\prime} \leq \operatorname{dim} \pi=8$, a contradiction.

Now $\psi \in I^{3} W_{q} F$ and $\psi_{L} \in I^{4} W_{q} L$. Therefore, Theorem 4.7 yields that there exists $d \in F$ with $\psi \equiv\langle\langle a, b, d]] \bmod I^{4} W_{q} F$.

Consider first the case $s=5$. Now $\langle 1, a, b, a b\rangle \prec\langle\langle a, b, d]]$ and $\langle 1, a, b, a b\rangle \prec$ $\rho$. Hence, $i_{\mathrm{ti}}(\langle\langle a, b, d]] \perp \rho)=i_{W}(\langle\langle a, b, d]] \perp \rho) \geq \operatorname{dim}\langle 1, a, b, a b\rangle=4$. Thus, $\langle\langle a, b, d]] \perp \rho \cong \rho^{\prime \prime} \perp(4 \times \mathbb{H})$ with $\rho^{\prime \prime}$ nonsingular and $\operatorname{dim} \rho^{\prime \prime}=10 . \quad \rho^{\prime \prime} \perp \sigma \sim$ $\langle\langle a, b, d]] \perp \rho \perp \sigma \sim\langle\langle a, b, d]] \perp \sigma \sim \sigma$, which shows for dimension reasons that $\rho^{\prime \prime}$ is an n.s.c. of $\sigma$. Furthermore, $q:=\varphi_{r} \perp \eta_{r} \perp \rho^{\prime} \in I^{4} W_{q} F$ and $2^{4}>2 s=10$. Lemma 6.7 implies that $q$ is similar to a Pfister form of which $\varphi \cong \varphi_{r} \perp \sigma$ is a Pfister neighbor.

Suppose now that $s=4$. Let $E=F(\sigma)$. Similarly as above, one shows that $\sigma^{\prime \prime}=\left(\sigma_{E}\right)_{\text {an }}$ is of dimension 2 or 3 , that the nondefective part $\varphi^{\prime \prime}$ of $\varphi_{E}$ is of type $\left(r, s^{\prime \prime}\right)$ with $s^{\prime \prime}=\operatorname{dim} \sigma^{\prime \prime}$, and that $\psi_{E(\varphi)} \sim 0$ and therefore $\psi_{E\left(\varphi^{\prime \prime}\right)} \sim 0$. Since $\psi \equiv$ $\langle\langle a, b, d]] \bmod I^{4} W_{q} F$, this implies $\langle\langle a, b, d]]_{E\left(\varphi^{\prime \prime}\right)} \equiv 0 \bmod I^{4} W_{q} E\left(\varphi^{\prime \prime}\right)$ and thus, by the Arason Pfister Hauptsatz, $\langle\langle a, b, d]]_{E\left(\varphi^{\prime \prime}\right)} \sim 0$. If $\langle\langle a, b, d]]_{E}$ is anisotropic, then by the domination theorem, there exists $y \in E^{*}$ with $y \varphi^{\prime \prime} \prec\langle\langle a, b, d]]$, hence $2 r+2 s^{\prime \prime} \leq \operatorname{dim}\langle\langle a, b, d]]=8$, a contradiction as $10 \leq 2 r+2 s^{\prime \prime}$. Hence, $\langle\langle a, b, d]]_{E} \sim 0$. If $\langle\langle a, b, d]]$ is anisotropic, then by the domination theorem and using the fact that both $\sigma$ and $\langle\langle a, b, d]]$ represent 1 , we get $\sigma \prec\langle\langle a, b, d]]$. This is clearly also true if $\langle\langle a, b, d]]$ is hyperbolic. In any case, similarly as above, there exists an n.s.c. $\rho^{\prime \prime}$ of $\sigma$ with $\rho \perp\langle\langle a, b, d]] \sim \rho^{\prime \prime}$ and $\sigma \prec \rho^{\prime \prime}$. Now, by putting $q:=\varphi_{r} \perp \eta_{r} \perp \rho^{\prime \prime}$, we have $q \in I^{4} W_{q} F$ and we conclude as before.

\section{Forms OF NONDEFECTIVE HEIGHT 1}

Let $q$ be an anisotropic form of type $(r, s)$ which is not totally singular, i.e., $r \geq 1$. Recall that $q$ is of nondefective height 1 if $\left(q_{F(q)}\right)$ an is totally singular, or in other words, by Lemmas 2.2 and $4.1\left(q_{F(q)}\right)_{\text {an }} \cong \mathrm{ql}(q)_{F(q)}$. Equivalently, $h_{\mathrm{nd}}(q)=1$ if and only if $i_{W}\left(q_{F(q)}\right)=r$. (In Knebusch's terminology [25], such a form is said to be of height 1.) Note that anisotropic forms of nondefective height 1 have, therefore, the property that their anisotropic part over their own function

field is defined over the base field, thus we can use our results from the previous section.

Example 7.1. Let $q$ be anisotropic of type $(1, s)$. Then clearly, $h_{\mathrm{nd}}(q)=1$ (Lemma 4.1).

Example 7.2. Let $q$ be an anisotropic Pfister neighbor of type $(r, s)$. Let $n$ be such that $2^{n-1}<\operatorname{dim} q=2 r+s \leq 2^{n}$. Then $q$ is a Pfister neighbor of some $n$ fold Pfister form and necessarily $2 r+2 s \leq 2^{n}$. The complement $q^{c}$ has dimension $\operatorname{dim} q^{c}=2^{n}-2 r-s$ and we have $\mathrm{ql}(q) \cong \mathrm{ql}\left(q^{c}\right)$ and $\left(q_{F(q)}\right)_{\text {an }} \cong q_{F(q)}^{c}$ by Proposition 
6.1. It follows immediately that $h_{\mathrm{nd}}(q)=1$ if and only if $2 r+2 s=2^{n}$ if and only if $\mathrm{ql}(q)=q^{c}$, i.e., $q$ is a close Pfister neighbor in Knebusch's terminology.

Example 7.3. We recall the construction in Example 6.4 of forms of nondefective height 1 which are not Pfister neighbors. Let $\psi$ be a Pfister neighbor of type $\left(r^{\prime}, s^{\prime}\right)$ of a Pfister form $\pi \cong\left\langle\left\langle a_{1}, \cdots, a_{n} ; b\right]\right]$, let $\tau \cong\left\langle\left\langle a_{1}, \cdots, a_{m}\right\rangle\right\rangle$ with $m \geq n+1$, and suppose that $\pi \perp x \tau, x \in F^{*}$, is anisotropic. Suppose furthermore that $2 r^{\prime}+2 s^{\prime}=2^{n+1}$ so that $\psi$ is a close Pfister neighbor of $\pi$. Let $q=\psi \perp x \tau$. Then $\mathrm{ql}(q)=\psi^{c} \perp x \tau, q$ is not a Pfister neighbor, and $h_{\text {nd }}(q)=1$ (see Example 6.4).

Lemma 7.4. Suppose that $q$ is anisotropic of type $(r, s), r \geq 2$. If there exists an integer $n \geq 1$ such that $r+s<2^{n}<2 r+s$, then $h_{\mathrm{nd}}(q) \geq 2$.

Proof. Suppose that $h_{\text {nd }}(q)=1$. Let $V$ be the underlying vector space of $q$, and let $W \subset V$ be a subspace with $\operatorname{dim} W=r+s+1$ and $q^{\prime}=\left.q\right|_{W}$. By assumption, $i_{W}\left(q_{F(q)}\right)=r$. Hence, $V \otimes F(q)$ contains a totally isotropic subspace (with respect to $\left.q_{F(q)}\right)$ of dimension $r$ which intersects with $W \otimes F(q)$ as $r+\operatorname{dim} W>\operatorname{dim} V$. In particular, $q_{F(q)}^{\prime}$ is isotropic. But $q^{\prime} \prec q$ and therefore, $q^{\prime}$ is anisotropic over $F$, and $\operatorname{dim} q^{\prime} \leq 2^{n}<\operatorname{dim} q$, a contradiction to Theorem 4.4

We now come to the classification of forms of nondefective height 1 of type $(r, s)$ with $r \geq 2$ and small values of $s$ (recall that the case $r=1$ is trivial by Example 7.1).

Theorem 7.5. Let $q$ be anisotropic of type $(r, s), r \geq 2$. Suppose that $s \leq 4$ or that $s=5$ and there exist $u, v, w \in F^{*}$ such that $\mathrm{ql}(q)$ is similar to $\langle\langle u, v\rangle\rangle \perp\langle w\rangle$. Then $h_{\mathrm{nd}}(q)=1$ if and only if $q$ belongs to one of the following (mutually exclusive) classes of forms:

(i) There exists an integer $n \geq 1$ such that $r+s=2^{n}$ and $q$ is a Pfister neighbor.

(ii) $(r, s)=(2,4)$ and there exist $a, b, c, d, e \in F^{*}$ such that $q \cong d\langle\langle a, b]] \perp$ $e\langle\langle a, c\rangle\rangle$.

Proof. If $q$ is as in (i), then $q$ is in fact a close Pfister neighbor of some $(n+1)$-fold Pfister form since $2 r+2 s=2^{n+1}$, and we have that $h_{\mathrm{nd}}(q)=1$ by Example 7.2

If $q$ is as in (ii), then $h_{\text {nd }}(q)=1$ by Example 7.3 Note that in this case, $q$ cannot be a Pfister neighbor as $\operatorname{dim} q=8$ and $q$ is not similar to a 3 -fold Pfister form.

Now assume that $h_{\text {nd }}(q)=1$. Then $\left(q_{F(q)}\right)_{\text {an }} \cong \mathrm{ql}(q)_{F(q)}$. If $2 r>s$, then by Theorem 6.6 $q$ is a Pfister neighbor, say, of an $(n+1)$-fold Pfister form, and we have $\left(q_{F(q)}\right)_{\text {an }} \cong q_{F(q)}^{c}$ by Proposition 6.1. Hence, $\operatorname{dim} q^{c}=\operatorname{dim} \mathrm{ql}(q)$. Now $\mathrm{ql}(q) \subset q^{c}$ and thus $q^{c} \cong \mathrm{ql}(q)$. This readily yields that $q$ is a Pfister neighbor of codimension $s$, i.e., $\operatorname{dim} q=2 r+s=2^{n+1}-s$, and we get $r+s=2^{n}$.

Since $s \leq 5$ and $r \geq 2$, the only cases which are not yet covered are $(r, s) \in$ $\{(2,4),(2,5)\}$. The case $(r, s)=(2,5)$ cannot occur by Lemma 7.4.

Let us finally assume that $(r, s)=(2,4)$ and write (after possibly scaling) $q \cong$ $\varphi \perp\langle 1, u, v, w\rangle$ with $\varphi$ nonsingular of dimension 4 and $u, v, w \in F^{*}$. By Proposition 8.6, the anisotropy of $\langle 1, u, v, w\rangle$ yields two possible cases: Either $\left[F^{2}(u, v, w)\right.$ : $\left.F^{2}\right]=8$, or $\left[F^{2}(u, v, w): F^{2}\right]=4$, and in this latter case one has in fact $\langle 1, u, v, w\rangle \cong$ $\langle 1, u, v, u v\rangle$.

Suppose first that $\left[F^{2}(u, v, w): F^{2}\right]=8$ and let $\left.K=F(\langle u, v\rangle\rangle\right)$. It follows readily from Corollary 8.14 that $\langle\langle u, w\rangle\rangle_{K}$ is anisotropic, hence also $\langle 1, u, w\rangle_{K}$. Now 
$\langle 1, u, v\rangle_{K}$ is isotropic (Proposition [8.9), hence $\left(\langle 1, u, v, w\rangle_{K}\right)_{\text {an }} \cong\langle 1, u, w\rangle_{K}$. Let $q^{\prime} \cong \varphi \perp\langle 1, u, w\rangle$. Then $q_{K} \sim q_{K}^{\prime}, q_{K}^{\prime}$ is nondefective, and $K(q)$ is a purely transcendental extension of $K\left(q^{\prime}\right)$. Now $q_{F(q)} \sim\langle 1, u, v, w\rangle$. Hence,

$$
\langle 1, u, v, w\rangle_{K(q)} \sim q_{K(q)} \sim q_{K(q)}^{\prime} \sim\langle 1, u, w\rangle_{K(q)}
$$

and thus $q_{K\left(q^{\prime}\right)}^{\prime} \sim\langle 1, u, w\rangle_{K\left(q^{\prime}\right)}$.

If $q_{K}^{\prime}$ is anisotropic, then $q_{K}^{\prime}$ is of type $(r, s-1)=(2,3)$ and $h_{\mathrm{nd}}\left(q_{K}^{\prime}\right)=1$ which, by the above, implies that $q_{K}^{\prime}$ is a Pfister neighbor, necessarily of some 3 -fold, which is impossible as $2 r+2(s-1)=10>8$. If $\left(q_{K}^{\prime}\right)_{\text {an }}$ is of type $(1,3)$, then $i_{W}\left(q_{K}^{\prime}\right)=1$ and $K\left(q^{\prime}\right) / K$ is purely transcendental. This implies that the 5-dimensional form $\left(q_{K}^{\prime}\right)_{\text {an }}$ stays anisotropic over $K\left(q^{\prime}\right)$, impossible as $q_{K\left(q^{\prime}\right)}^{\prime} \sim\langle 1, u, w\rangle_{K\left(q^{\prime}\right)}$. Hence, $q_{K}^{\prime}$ is of type $(0,3)$, i.e., $q_{K}^{\prime} \sim\langle 1, u, w\rangle_{K}$.

Now by assumption, $q_{F(q)} \sim\langle 1, u, v, w\rangle_{F(q)}$, and by Lemma 6.8, there exists an n.s.c. $\psi$ of $q$ with $\operatorname{dim} \psi=2 r+2 s=12$ and $\psi \in I^{3} W_{q} F$. By Lemma 3.9, there exists an n.s.c. $\tau$ over $K$ of $\langle 1, u, v, w\rangle_{K} \cong\langle 1, u, w, 0\rangle_{K}$ such that $\psi_{K} \sim \tau \in I^{3} W_{q} K$. Since $\operatorname{dim} \tau=8$ and since $\tau$ is isotropic, it follows from the Arason-Pfister Hauptsatz that $\psi_{K} \sim \tau \sim 0$. By Theorem 4.7 there exists $x \in F^{*}$ with $\psi \equiv\langle\langle u, v, x]] \bmod I^{4} W_{q} F$. Now $i_{W}(\psi \perp\langle\langle u, v, x]]) \geq 3$, hence $\operatorname{dim}(\psi \perp\langle\langle u, v, x]])_{\text {an }} \leq 12+8-6=14<16$, and again by the Arason-Pfister Hauptsatz, $\psi \perp\langle\langle u, v, x]] \sim 0$. Hence,

$$
\psi \perp\langle 1, u, v, w\rangle \sim q \sim\langle\langle u, v, x]] \perp\langle 1, u, v, w\rangle \sim u v[1, x] \perp\langle 1, u, v, w\rangle,
$$

and by comparing dimensions, we conclude that $q$ is isotropic, a contradiction.

Thus, we have $\left[F^{2}(u, v, w): F^{2}\right]=4$ and $q \cong \varphi \perp\langle 1, u, v, u v\rangle$. Again by Lemma 6.8, there exist $x, y, z \in F$ such that $\varphi \perp[1, x] \perp[u, y] \perp[v, z] \perp[u v, 0] \in I^{3} W_{q} F$. Now there are no anisotropic forms of dimension 10 in $I^{3} W_{q} F$ (11] pp. 129130]), and thus, by the Arason-Pfister Hauptsatz, there exists $\pi \in G P_{3} F$ such that $\varphi \perp[1, x] \perp[u, y] \perp[v, z] \sim \pi$. Hence,

$$
\varphi \perp[1, x] \perp[u, y] \perp[v, z] \perp \pi \perp\langle 1, u, v, u v\rangle \sim q \perp \pi \sim\langle 1, u, v, u v\rangle .
$$

This implies that $(q \perp \pi)_{\text {an }} \cong\langle 1, u, v, u v\rangle$. Now $\operatorname{dim}(q \perp \pi)=16$, therefore $i_{W}(q \perp \pi)=6$, and by Proposition 3.11, there exists a form $\eta$ such that $\operatorname{dim} \eta=6$, $\eta \prec \pi, \eta \prec q$. In particular, $\eta$ is a 6 -dimensional Pfister neighbor, therefore of type $(3,0)$ or $(2,2)$. But $q$ is of type $(2,4)$ and $\eta \prec q$, hence $\eta$ is of type $(2,2)$. Then there exist $a, b, d, e \in F^{*}$ such that $\eta \cong d\langle\langle a, b]] \perp e\langle 1, a\rangle$ (see [28, Prop. 3.2]). Since $\eta \prec q$, it follows that there exist $c, f \in F^{*}$ such that $q \cong d\langle\langle a, b]] \perp e\langle 1, a, c, f\rangle$. Comparing quasi-linear parts, we obtain $e\langle 1, a, c, f\rangle \cong\langle 1, u, v, u v\rangle$. Propositions 8.5 and 8.6 readily imply that $\langle 1, u, v, u v\rangle \cong\langle 1, a, c, f\rangle \cong\langle 1, a, c, a c\rangle$.

\section{Totally SINGUlar FORMS AND QUASI-PFISTER NEIGHBORS}

In Theorem 6.6 in the case $s=5$, we have considered only the case of totally singular forms similar to forms of type $\langle\langle a, b\rangle\rangle \perp\langle c\rangle$. In characteristic $\neq 2$, forms similar to such diagonal forms are exactly the 5-dimensional Pfister neighbors, so it seems natural to ask whether there is a meaningful notion of totally singular Pfister neighbors in characteristic 2 .

The purpose of this section is to develop some basic notions of a theory of totally singular forms in a way which is as much independent of the theory of nonsingular forms as possible and yet reflects some analogous properties, including a notion analogous to that of Pfister neighbors. 
Some basic properties have been mentioned before as they have been used in the proofs of our results. Let us recall some of them to keep this section as self-contained as possible. (See also Lemma [2.2] and the remarks preceding it.)

Recall that the anisotropic part of a totally singular form $\sigma=\left\langle a_{1}, \cdots, a_{n}\right\rangle$ is uniquely determined by the $F^{2}$-vector space spanned by $\left\{a_{1}, \cdots, a_{n}\right\}$ inside $F$, and this $F^{2}$-vector space is nothing but $D_{F}(\sigma) \cup\{0\}$. Furthermore, $i_{\mathrm{ql}}(\sigma)=i_{\mathrm{ti}}(\sigma)=$ $\operatorname{dim} \sigma-\operatorname{dim}_{F^{2}}\left(D_{F}(\sigma) \cup\{0\}\right)$. This immediately implies

\section{Proposition 8.1. $\quad$ (i) There is a one-to-one correspondence}

$$
\begin{aligned}
\left\{\begin{array}{l}
\text { isometry classes of } \\
\text { totally singular forms }
\end{array}\right\} & \rightleftharpoons\left\{\begin{array}{l}
\text { finite-dimensional } \\
\left.F^{2} \text {-vector spaces inside } F\right\} \times \mathbb{N}_{0}
\end{array}\right. \\
\sigma & \rightarrow\left(V, \operatorname{dim} \sigma-\operatorname{dim}_{F^{2}} V\right) \text { for } V=D_{F}(\sigma) \cup\{0\} \\
\left\langle a_{1}, \cdots, a_{n}\right\rangle \perp m \times\langle 0\rangle & \leftarrow(V, m) \text { for an } F^{2} \text {-basis }\left\{a_{1}, \cdots, a_{n}\right\} \text { of } V .
\end{aligned}
$$

(ii) $\varphi=\left\langle a_{1}, \cdots, a_{n}\right\rangle \subset \psi=\left\langle b_{1}, \cdots, b_{m}\right\rangle$ if and only if $i_{\mathrm{ti}}(\psi) \geq i_{\mathrm{ti}}(\varphi)$ and the sub- $F^{2}$-vector space inside $F$ spanned by $\left\{a_{1}, \cdots, a_{n}\right\}$ is contained in the sub- $F^{2}$-vector space inside $F$ spanned by $\left\{b_{1}, \cdots, b_{n}\right\}$ if and only if $i_{\mathrm{ti}}(\psi) \geq i_{\mathrm{ti}}(\varphi)$ and $D_{F}(\varphi) \cup\{0\} \subset D_{F}(\psi) \cup\{0\}$.

(iii) (Cf. Lemma [2.2) Let $\varphi=\left\langle a_{1}, \cdots, a_{n}\right\rangle$. If $E / F$ is any field extension, then there exist (after possibly reindexing) $0 \leq m \leq n$ such that $\left(\varphi_{E}\right)_{\mathrm{an}} \cong$ $\left\langle a_{1}, \cdots, a_{m}\right\rangle$.

Recall that over a field $K$ of characteristic $\neq 2$, the norm group of a quadric $Q$ associated to a (nonsingular) quadratic form $\varphi$ is given by the subgroup of $K^{*}$ generated by elements $a b$ with $a, b \in K^{*}$ represented by $\varphi$ (see [13, Lemme 2.2]). In analogy to this we make the following definition:

Definition 8.2. Let $\sigma$ be a totally singular form over $F$. The norm field of $\sigma$ over $F$, denoted by $N_{F}(\sigma)$, is defined to be the field $F^{2}\left(a b \mid a, b \in D_{F}(\sigma)\right)$. The norm degree of $\sigma$ over $F$ is defined to be $\operatorname{ndeg}_{F}(\sigma)=\left[N_{F}(\sigma): F^{2}\right]$.

The proof of the following lemma is easy and left to the reader.

Lemma 8.3. Let $\sigma \cong\left\langle a_{1}, \cdots, a_{n}\right\rangle$ be a nonzero totally singular form with $a_{1} \neq 0$. Then, for each $a \in F^{*}$, we have $N_{F}(a \sigma)=N_{F}(\sigma)=F^{2}\left(a_{1} a_{2}, \cdots, a_{1} a_{n}\right)$.

This implies, in particular, that the norm field and the norm degree are invariants of the similarity class of a totally singular form.

This lemma also readily implies

Corollary 8.4. Let $\sigma$ be a nonzero totally singular form and let $K / F$ be a field extension. Let $b_{1}, \cdots, b_{m} \in F$ be such that $N_{F}(\sigma)=F^{2}\left(b_{1}, \cdots, b_{m}\right)$. Then $N_{K}\left(\sigma_{K}\right)=K^{2}\left(b_{1}, \cdots, b_{m}\right)$.

We now come to the characterization of quasi-Pfister forms.

Proposition 8.5. $\quad$ (i) Let $q=\left\langle\left\langle a_{1}, \cdots, a_{n}\right\rangle\right\rangle$ be a quasi-Pfister form over $F$. Then $N_{F}(q)=F^{2}\left(a_{1}, \cdots, a_{n}\right)=D_{F}^{*}(q) \cup\{0\}$. In particular, if $x \in D_{F}^{*}(q)$, then $q \cong x q$.

(ii) Conversely, let $q$ be a nonzero totally singular form such that $q \cong x q$ for each $x \in D_{F}^{*}(q)$. Then $q_{\text {an }}$ is a quasi-Pfister form.

(iii) An anisotropic totally singular form $q$ is similar to a quasi-Pfister form if and only if $\operatorname{dim} q=\operatorname{ndeg}_{F}(q)$. 
(iv) There exists a natural bijection between anisotropic $n$-fold quasi-Pfister forms and purely inseparable extensions of $F^{2}$ of degree $2^{n}$ inside $F$ which is given by $\left\langle\left\langle a_{1}, \cdots, a_{n}\right\rangle\right\rangle \leftrightarrow F^{2}\left(a_{1}, \cdots, a_{n}\right)$.

Proof. (i) Let $q=\left\langle\left\langle a_{1}, \cdots, a_{n}\right\rangle\right\rangle$ with $a_{i} \in F^{*}$. Clearly, $N_{F}(q)=F^{2}\left(a_{1}, \cdots, a_{n}\right)$. Also, we obviously have $F^{2}\left[a_{1}, \cdots, a_{n}\right]=D_{F}(q) \cup\{0\} \subset N_{F}(q)$. Since the $a_{i}$ are algebraic over $F^{2}$, we have $D_{F}(q) \cup\{0\}=N_{F}(q)$. It follows readily that the coefficients of the diagonal forms $q$ and $x q, x \in N_{F}(q)^{*}=D_{F}^{*}(q)$ span the same subvector space of the $F^{2}$-vector space $F$, namely $N_{F}(q)$. Hence, $q \cong x q$.

(ii) Since $\langle 0\rangle \cong x\langle 0\rangle$ for each $x \in F$, we clearly may assume by Lemma 2.6 that $q$ is anisotropic. $R=D_{F}(q) \cup\{0\}$ is an additive subgroup of $F$, and the roundness of $q$ implies that $D_{F}(q)$ is closed under multiplication. Hence, $R$ is a finite-dimensional $F^{2}$-algebra inside the field $F$ and one obviously has $\operatorname{dim}_{F^{2}}(R)=$ $\operatorname{dim} q$. In particular, $R$ is a field. Let $q=\left\langle a_{1}, \cdots, a_{m}\right\rangle$ and let $n \leq m$ be minimal such that, after possibly reindexing, $F^{2}\left(a_{1}, \cdots, a_{m}\right)=F^{2}\left(a_{1}, \cdots, a_{n}\right)$. Similarly, as in part (i), we get $R=F^{2}\left(a_{1}, \cdots, a_{n}\right)$ which, by the minimality of $n$, yields $m=$ $\operatorname{dim}_{F^{2}} R=2^{n}=\operatorname{dim} q$. Now $\left\{a_{1}, \cdots, a_{m}\right\}$ is an $F^{2}$-basis of $R$, as is $\left\{\prod_{i \in I} a_{i} \mid I \subset\right.$ $\{1, \cdots, n\}\}$. It follows readily that $q \cong\left\langle\left\langle a_{1}, \cdots, a_{n}\right\rangle\right\rangle$.

(iii) Let $\operatorname{ndeg}_{F}(q)=2^{n}$ and $a_{1}, \cdots, a_{n}$ with $N_{F}(q)=F^{2}\left(a_{1}, \cdots, a_{n}\right)$. Suppose that $\operatorname{dim} q=\operatorname{ndeg}(q)$. We may assume that $q \cong\langle 1, \cdots\rangle$ and thus $V=D_{F}(q) \cup\{0\} \subset$ $N_{F}(q)=D_{F}\left(\left\langle\left\langle a_{1}, \cdots, a_{n}\right\rangle\right\rangle\right) \cup\{0\}$. By Proposition 8.1](ii), we get $q \subset\left\langle\left\langle a_{1}, \cdots, a_{n}\right\rangle\right\rangle$ and hence $q \cong\left\langle\left\langle a_{1}, \cdots, a_{n}\right\rangle\right\rangle$ for dimension reasons.

The converse is clear.

(iv) is obvious and left to the reader.

Proposition 8.6. Let $\sigma$ be a nonzero totally singular form.

(i) There exists an $m \geq 0$ such that $\operatorname{ndeg}_{F}(\sigma)=2^{m}$.

(ii) With $m$ as in (i), we have $m+1 \leq \operatorname{dim} \sigma_{\text {an }} \leq 2^{m}$.

(iii) With $m$ as in (i) and if $a_{1}, \cdots, a_{m} \in F^{*}$ are chosen such that $N_{F}(\sigma)=$ $F^{2}\left(a_{1}, \cdots, a_{m}\right)$, then for each $a \in D_{F}^{*}(\sigma)$ we have $a \sigma_{\text {an }} \subset\left\langle\left\langle a_{1}, \cdots, a_{m}\right\rangle\right\rangle$. Furthermore, if $b_{1}, \cdots, b_{m} \in F^{*}$ are such that $a \sigma_{\text {an }} \subset\left\langle\left\langle b_{1}, \cdots, b_{m}\right\rangle\right\rangle$, then $\left\langle\left\langle a_{1}, \cdots, a_{m}\right\rangle\right\rangle \cong\left\langle\left\langle b_{1}, \cdots, b_{m}\right\rangle\right\rangle$, and this $m$-fold quasi-Pfister form is anisotropic.

Proof. We may assume that $\sigma$ is anisotropic and $\sigma \cong\left\langle 1, a_{1}, \cdots, a_{n}\right\rangle$. Hence, $N_{F}(\sigma)=F^{2}\left(a_{1}, \cdots, a_{n}\right)$. Let $m \leq n$ be minimal such that (after reindexation) $N_{F}(\sigma)=F^{2}\left(a_{1}, \cdots, a_{m}\right)$. It is clear that $2^{m}=\operatorname{ndeg}(\sigma)$, which shows (i). To prove (ii), we note that the anisotropy of $\sigma$ implies that the $\left\{1, a_{1}, \cdots, a_{n}\right\} \subset N_{F}(\sigma)$ are $F^{2}$-linearly independent and can therefore be extended to an $F^{2}$-basis of the extension $N_{F}(\sigma) / F^{2}$, which immediately yields that $\sigma \subset\left\langle\left\langle a_{1}, \cdots, a_{m}\right\rangle\right\rangle$. Hence, $m+1 \leq \operatorname{dim} \sigma \leq 2^{m}$. Part (iii) follows readily from Proposition 8.5)(iv).

Proposition 8.7. Let $\sigma$ be anisotropic totally singular, $\operatorname{ndeg}_{F}(\sigma)=2^{m}$ and let $a_{1}, \cdots, a_{m} \in F^{*}$ be such that $N_{F}(\sigma)=F^{2}\left(a_{1}, \cdots, a_{m}\right)$. Let $K / F$ be any field extension such that $\sigma_{K}$ is isotropic. Then $\operatorname{ndeg}_{K}\left(\sigma_{K}\right)=2^{\ell}<2^{m}$ and there exist $\left\{a_{i_{1}}, \cdots, a_{i_{\ell}}\right\} \subset\left\{a_{1}, \cdots, a_{m}\right\}$ such that $N_{K}\left(\sigma_{K}\right)=K^{2}\left(a_{i_{1}}, \cdots, a_{i_{\ell}}\right)$. In particular, for each $a \in D_{K}^{*}(\sigma)$, we have $a\left(\sigma_{K}\right)_{\text {an }} \subset\left\langle\left\langle a_{i_{1}}, \cdots, a_{i_{\ell}}\right\rangle\right\rangle$.

Proof. After scaling, we may assume that $1 \in D_{F}(\sigma)$. By Proposition 8.6 (iii), $\sigma \subset\left\langle\left\langle a_{1}, \cdots, a_{m}\right\rangle\right\rangle$. Now $\sigma_{K}$ is isotropic, hence also $\left\langle\left\langle a_{1}, \cdots, a_{m}\right\rangle_{K}\right.$. By Proposition 8.5](iv), this implies that $\left[K^{2}\left(a_{1}, \cdots, a_{m}\right): K^{2}\right]=2^{\ell}<2^{m}$. Clearly, we may 
find $\left\{a_{i_{1}}, \cdots, a_{i_{\ell}}\right\} \subset\left\{a_{1}, \cdots, a_{m}\right\}$ with $K^{2}\left(a_{1}, \cdots, a_{m}\right)=K^{2}\left(a_{i_{1}}, \cdots, a_{i_{\ell}}\right)$. Now $N_{K}\left(\sigma_{K}\right)=K^{2}\left(a_{1}, \cdots, a_{m}\right)$. All this, together with Proposition 8.6)(iii) implies the desired result.

Definition 8.8. A totally singular form $\sigma$ is called a quasi-Pfister neighbor if there exists $a \in F^{*}$ and an anisotropic $m$-fold quasi-Pfister form $\pi(m \geq 0)$ such that $a \sigma \subset \pi$ and $2 \operatorname{dim} \sigma>\operatorname{dim} \pi=2^{m}$. In this situation, $\sigma$ is said to be a quasi-Pfister neighbor of $\pi$.

Proposition 8.9. (i) If $\sigma$ is a quasi-Pfister neighbor of the anisotropic $m$-fold quasi-Pfister form $\pi$, then $N_{F}(\sigma)=N_{F}(\pi)$. In particular, if $\sigma$ is also a quasi-Pfister neighbor of another anisotropic $m$-fold quasi-Pfister form $\tau$, then $\pi \cong \tau$.

(ii) A totally singular form $\sigma$ is a quasi-Pfister neighbor if and only if $\sigma$ is anisotropic and $2 \operatorname{dim} \sigma>\operatorname{ndeg}_{F}(\sigma)$.

(iii) Let $\sigma$ be a quasi-Pfister neighbor of the anisotropic m-fold quasi-Pfister form $\pi, m \geq 1$. Let $E / F$ be a field extension. Then $\sigma_{E}$ is isotropic iff $\pi_{E}$ is isotropic.

Proof. (i) Clearly, $N_{F}(\sigma) \subset N_{F}(\pi)$. Hence $\operatorname{ndeg}_{F}(\sigma) \leq \operatorname{ndeg}_{F} \pi=2^{m}$. Since $\sigma$ is anisotropic of dimension $>2^{m-1}$, it follows from Proposition 8.6 that $\operatorname{ndeg}_{F}(\sigma)=$ $2^{m}$ and thus $N_{F}(\sigma)=N_{F}(\pi)$. Now if $\sigma$ is also a quasi-Pfister neighbor of $\tau$, then $N_{F}(\pi)=N_{F}(\tau)$ and $\pi \cong \tau$ by Proposition 8.5 .

(ii) If $\sigma$ is a quasi-Pfister neighbor, say, of the anisotropic $m$-fold quasi-Pfister form $\pi$, then by (i) we have

$$
2 \operatorname{dim} \sigma>\operatorname{dim} \pi=2^{m}=\operatorname{ndeg}_{F}(\pi)=\operatorname{ndeg}_{F}(\sigma)
$$

Conversely, let $\sigma$ be anisotropic and $\operatorname{ndeg}_{F}(\sigma)=2^{m}$. Then there exist $a_{1}, \cdots, a_{m}$ $\in F^{*}$ with $N_{F}(\sigma)=F^{2}\left(a_{1}, \cdots, a_{m}\right)$. Then $\pi=\left\langle\left\langle a_{1}, \cdots, a_{m}\right\rangle\right\rangle$ is anisotropic (Proposition 8.5) and for each $a \in D_{F}^{*}(\sigma)$, we have $a \sigma \subset \pi$ (Proposition 8.6). Now $2 \operatorname{dim} \sigma>\operatorname{ndeg}_{F}(\sigma)=2^{m}=\operatorname{dim} \pi$ implies that $\sigma$ is a Pfister neighbor of $\pi$.

(iii) Clearly, the isotropy of $\sigma_{E}$ implies that of $\pi_{E}$. Now the definition of a quasiPfister neighbor and its associated quasi-Pfister form imply by Proposition 8.6 that $N_{E}\left(\sigma_{E}\right)=N_{E}\left(\pi_{E}\right)$. Hence $\operatorname{ndeg}_{E}\left(\sigma_{E}\right)=\operatorname{ndeg}_{E}\left(\pi_{E}\right)$. Now $\pi_{E}$ being isotropic implies that $\operatorname{ndeg}_{E}\left(\pi_{E}\right) \leq 2^{m-1}$, hence $\operatorname{dim}\left(\sigma_{E}\right)_{\text {an }} \leq 2^{m-1}<\operatorname{dim} \sigma$.

Recall that in characteristic $\neq 2$, the splitting pattern of a quadratic form is the increasing sequence of Witt indices of this form over the fields in a generic splitting tower (see, e.g., [19], 20], 17]). In characteristic 2, it it more reasonable to define a standard splitting pattern of a (possibly singular) form to be the sequence of pairs $(r, s)$ denoting the types of the anisotropic parts of this form over the fields in the standard splitting tower (see, e.g., [29]). For totally singular forms, this just means the sequence of the dimensions of the anisotropic parts over the fields in the standard splitting tower (cf. subsection 4.2). Hence, if $F=F_{0} \subset F_{1} \subset \cdots \subset$ $F_{h-1} \subset F_{h}$ denotes the standard splitting tower of the nonzero totally singular form $\sigma$ of height $h$, then the standard splitting pattern is given by $\left(s_{0}, \cdots, s_{h-1}, s_{h}\right)$ with $s_{i}=\operatorname{dim}\left(\sigma_{F_{i}}\right)_{\text {an }}$.

The following result is a more precise version of Proposition 8.7 in the case where $K=F(\sigma)$. 
Lemma 8.10. Let $\sigma \cong\langle 1\rangle \perp \sigma^{\prime}$ be an anisotropic totally singular form of dimension $\geq 2$ and let $K=F(\sigma)$. Suppose that $\operatorname{ndeg}_{F}(\sigma)=2^{m}$ and let $b_{1}, \cdots, b_{m} \in F^{*}$ be such that $N_{F}(\sigma)=F^{2}\left(b_{1}, \cdots, b_{m}\right)$. Then $\operatorname{ndeg}_{K}\left(\sigma_{K}\right)=2^{m-1}, N_{K}\left(\sigma_{K}\right)=$ $K^{2}\left(b_{2}, \cdots, b_{m}\right)$. In particular, $\left(\sigma_{K}\right)_{\text {an }} \subset\left\langle\left\langle b_{2}, \cdots, b_{m}\right\rangle_{K}\right.$.

Proof. Since $1 \in D_{F}(\sigma)$, we have $N_{F}(\sigma)=F^{2}\left(a \mid a \in D_{F}\left(\sigma^{\prime}\right)\right)$. Hence, there exists $c_{1} \in D_{F}\left(\sigma^{\prime}\right)$ with $c_{1} \notin F^{2}\left(b_{2}, \cdots, b_{m}\right)$. Thus, $F^{2}\left(b_{1}, \cdots, b_{m}\right)=F^{2}\left(c_{1}, b_{2}, \cdots, b_{m}\right)$. Let $n=\operatorname{dim} \sigma^{\prime}$ and let $c_{2}, \cdots, c_{n} \in F^{*}$ such that $\sigma^{\prime} \cong\left\langle c_{1}, \cdots, c_{n}\right\rangle$. For $i \geq 2$, write $c_{i}=u_{i}+c_{1} v_{i}$ with $u_{i}, v_{i} \in F^{2}\left(b_{2}, \cdots, b_{m}\right)$.

Let $X_{1}, \cdots, X_{n}$ be variables and put $L=F\left(X_{1}, \cdots, X_{n}\right), d=\sum_{i=1}^{n} c_{i} X_{i}^{2}$. Then $K=L(\sqrt{d})$ and $K^{2}=L^{2}(d)$. If we put $r=X_{1}^{2}+\sum_{i=2}^{n} v_{i} X_{i}^{2}$ and $s=\sum_{i=2}^{m} u_{i} X_{i}^{2}$, then clearly $r, s \in L^{2}\left(b_{2}, \cdots, b_{m}\right), r \neq 0$ and $d=s+c_{1} r$. Hence, $L^{2}\left(b_{1}, \cdots, b_{m}\right)=$ $L^{2}\left(c_{1}, b_{2}, \cdots, b_{m}\right)=L^{2}\left(d, b_{2}, \cdots, b_{m}\right)=K^{2}\left(b_{2}, \cdots, b_{m}\right)$.

By Corollary 8.4 $N_{K}\left(\sigma_{K}\right)=K^{2}\left(b_{1}, \cdots, b_{m}\right)=L^{2}\left(d, b_{1}, \cdots, b_{m}\right)$, and thus, by the above, $N_{K}\left(\sigma_{K}\right)=K^{2}\left(b_{2}, \cdots, b_{m}\right)$.

Since $L^{2} / F^{2}$ is purely transcendental, we clearly have $2^{m}=\left[F^{2}\left(b_{1}, \cdots, b_{m}\right)\right.$ : $\left.F^{2}\right]=\left[L^{2}\left(b_{1}, \cdots, b_{m}\right): L^{2}\right]=\left[K^{2}\left(b_{2}, \cdots, b_{m}\right): L^{2}\right]$. Now $L^{2}(d)=K^{2}$ and $\left[K^{2}\right.$ : $\left.L^{2}\right]=2$. Thus, $\operatorname{ndeg}_{K}\left(\sigma_{K}\right)=\left[N_{K}\left(\sigma_{K}\right): K^{2}\right]=\left[K^{2}\left(b_{2}, \cdots, b_{m}\right): K^{2}\right]=2^{m-1}$.

Finally, $\left(\sigma_{K}\right)_{\text {an }} \subset\left\langle\left\langle b_{2}, \cdots, b_{m}\right\rangle\right\rangle_{K}$ follows from Proposition 8.6 and the fact that $1 \in D_{K}\left(\sigma_{K}\right)$.

It is now easy, to compute the standard splitting pattern for quasi-Pfister neighbors.

Theorem 8.11. Let $\sigma$ be an anisotropic totally singular form. Let $F=F_{0} \subset F_{1} \subset$ $\cdots \subset F_{h}$ be the standard splitting tower of $\sigma$ and let $\sigma_{i}=\left(\sigma_{F_{i}}\right)_{\text {an }}, s_{i}=\operatorname{dim} \sigma_{i}$. Let $n_{i}$ and $m$ be such that $2^{n_{i}}<s_{i} \leq 2^{n_{i}+1}$ and $\operatorname{ndeg}_{F}(\sigma)=2^{m}$. Let $b_{1}, \cdots, b_{m} \in F^{*}$ be such that $N_{F}(\sigma)=F^{2}\left(b_{1}, \cdots, b_{m}\right)$.

(i) For $1 \leq i \leq h$, we have $\operatorname{ndeg}_{F_{i}}\left(\sigma_{i}\right)=2^{m-i}, N_{F_{i}}\left(\sigma_{i}\right)=F_{i}^{2}\left(b_{1}, \cdots, b_{m-i}\right)$, and $\max \left\{m-i+1,2^{n_{i-1}}\right\} \leq \operatorname{dim} \sigma_{i} \leq 2^{m-i}$. In particular, $h(\sigma)=m$. Furthermore, for every $a \in D_{F}(\sigma)$, we have $a \sigma_{i} \subset\left\langle\left\langle b_{1}, \cdots, b_{m-i}\right\rangle\right\rangle_{F_{i}}$.

(ii) $\sigma$ is a Pfister neighbor iff $\left(s_{0}, s_{1}, \cdots, s_{h}\right)=\left(\operatorname{dim} \sigma, 2^{m-1}, 2^{m-2}, \cdots, 1\right)$. In this situation, $a \sigma_{i} \cong\left\langle\left\langle b_{1}, \cdots, b_{m-i}\right\rangle\right\rangle_{F_{i}}$ for every $a \in D_{F}(\sigma)$.

Proof. (i) $\operatorname{ndeg}_{F_{i}}\left(\sigma_{i}\right)=2^{m-i}, N_{F_{i}}\left(\sigma_{i}\right)=F_{i}^{2}\left(b_{1}, \cdots, b_{m-i}\right), m-i+1 \leq \operatorname{dim} \sigma_{i} \leq$ $2^{m-i}$ and $a \sigma_{i} \subset\left\langle\left\langle b_{1}, \cdots, b_{m-i}\right\rangle\right\rangle_{F_{i}}$ follow by induction from Proposition 8.6 and Lemma 8.10

Now $2^{n_{i-1}}<\operatorname{dim} \sigma_{i-1} \leq 2^{n_{i-1}+1}$. Theorem 4.4 yields that any subform of $\sigma_{i-1}$ of dimension $2^{n_{i-1}}$ stays anisotropic over $F_{i}$ and will therefore be also a subform of $\left(\left(\sigma_{i-1}\right)_{F_{i}}\right)_{\text {an }}=\sigma_{i}$. Hence $\operatorname{dim} \sigma_{i} \geq 2^{n_{i-1}}$.

$h(\sigma)=m$ now follows readily by induction on $m$ and the definition of height.

(ii) If $\sigma$ is a Pfister neighbor, then $2^{m-1}<\operatorname{dim} \sigma \leq 2^{m}=\operatorname{ndeg}_{F}(\sigma)$ by Proposition 8.9 and it follows immediately from (i) that $\sigma$ has standard splitting pattern $\left(\operatorname{dim} \sigma, 2^{m-1}, 2^{m-2}, \cdots, 1\right)$.

Conversely, if the standard splitting pattern is of that type, then $h(\sigma)=m$, hence $\operatorname{ndeg}_{F}(\sigma)=2^{m}$ and $\operatorname{dim} \sigma>2^{m-1}$. Hence, $\sigma$ is a quasi-Pfister neighbor by Proposition 8.9 .

The remaining statement follows immediately from (i) by comparing dimensions. 
We now give characterizations of quasi-Pfister neighbors of small dimension.

Proposition 8.12. Let $\sigma$ be an anisotropic totally singular form.

(i) If $\operatorname{dim} \sigma \leq 3$, then $\sigma$ is a quasi-Pfister neighbor.

(ii) If $\operatorname{dim} \sigma=2^{n}$, then $\sigma$ is a quasi-Pfister neighbor iff $\sigma$ is similar to a quasiPfister form.

(iii) If $\operatorname{dim} \sigma=5$, then $\sigma$ is a quasi-Pfister neighbor iff there exist $a, b, c \in F^{*}$ such that $\sigma$ is similar to $\langle 1, a, b, a b, c\rangle$.

(iv) If $\operatorname{dim} \sigma=6$, then $\sigma$ is a quasi-Pfister neighbor iff there exist $a, b, c \in F^{*}$ such that $\sigma$ is similar to $\langle 1, a, b, a b, c, a c\rangle$.

(v) If $\operatorname{dim} \sigma=7$, then $\sigma$ is a quasi-Pfister neighbor iff there exist $a, b, c \in F^{*}$ such that $\sigma$ is similar to $\langle a, b, c, a b, a c, b c, a b c\rangle$.

Proof. (i) and (ii) are easy and left to the reader. It is also obvious that anisotropic forms similar to forms of type $\langle 1, a, b, a b, c\rangle,\langle 1, a, b, a b, c, a c\rangle,\langle a, b, c, a b, a c, b c, a b c\rangle$ are quasi-Pfister neighbors. For the converse, let $\sigma$ be a quasi-Pfister neighbor with $5 \leq \operatorname{dim} \sigma \leq 7$.

The case $\operatorname{dim} \sigma=5$. After scaling, we may assume that $\sigma \cong\langle 1, p, q, r, s\rangle$, and by the definition of a quasi-Pfister neighbor and Proposition 8.6, we may furthermore assume that $\sigma \subset\langle\langle p, q, r\rangle\rangle$ with $\left[F^{2}(p, q, r): F^{2}\right]=8$. Then $0 \neq s=u+v$ with $v \in D_{F}(\langle 1, p, q, r\rangle) \cup\{0\}$ and $u=p q x^{2}+p r y^{2}+q r z^{2}+p q r t^{2}$ for suitable $x, y, z, t \in F$. If $u=0$, then $s \in D_{F}(\langle 1, p, q, r\rangle)$ and $\sigma$ is isotropic, a contradiction. Hence, $u \neq 0$ and we have $\langle 1, p, q, r, s\rangle \cong\langle 1, p, q, r, u\rangle$.

Suppose first that $t \neq 0$. Then, after multiplying $u$ by a square, we may assume $t=1$. Now $\langle 1, p, q, r\rangle \cong\left\langle 1, z^{2}+p, y^{2}+q, x^{2}+r\right\rangle$ and $\left(z^{2}+p\right)\left(y^{2}+q\right)\left(x^{2}+r\right)=u+w$ with $w \in D_{F}(\langle 1, p, q, r\rangle)$. Put $f=z^{2}+p, g=y^{2}+q, h=x^{2}+r$. Thus, we get $\sigma \cong\langle 1, p, q, r, u\rangle \cong\langle 1, p, q, r, u+w\rangle \cong\langle 1, f, g, h, f g h\rangle$. By putting $a=f g, b=f h$, $c=f g h$, we get $c \sigma \cong\langle 1, a, b, a b, c\rangle$.

Suppose now that $t=0$. Since $u \neq 0$, it suffices by symmetry to consider only the case $x \neq 0$. After scaling by a square, we may assume $x=1$. If $y=z=0$, then $\sigma \cong\langle 1, p, q, r, u\rangle \cong\langle 1, p, q, p q, r\rangle$ and we are done. Otherwise, again by symmetry, it suffices to consider the case $y \neq 0$. This time, we put $a=p+q(z / y)^{2}, b=q+r y^{2}$. Then $a b=u+w$ with $w \in F^{2}$ and we get

$$
\sigma \cong\langle 1, p, q, r, u\rangle \cong\langle 1, a, b, r, u\rangle \cong\langle 1, a, b, r, u+w\rangle \cong\langle 1, a, b, a b, r\rangle .
$$

The case $\operatorname{dim} \sigma=6$. By the 5-dimensional case, we may assume after scaling that $\sigma \cong\langle 1, p, q, p q, r, s\rangle \subset\langle\langle p, q, r\rangle\rangle$. Then we can write $s=u+r v$ with $u, v \in D_{F}(\langle\langle p, q\rangle\rangle) \cup\{0\}$, and the anisotropy of $\sigma$ implies $v \neq 0$. Hence, $\sigma \cong$ $\langle 1, p, q, p q, r, r v\rangle$. Also, we can write $v=x^{2}+w$ with $w \in D_{F}(\langle p, q, p q\rangle)$. Since $\left\langle r, r x^{2}\right\rangle$ is isotropic, we must have $w \neq 0$, and thus $w \in F^{2}(p, q) \backslash F^{2}$. But then there exists $t \in\{p, q\}$ with $F^{2}(p, q)=F^{2}(w, t)$ and we have $\langle\langle p, q\rangle\rangle \cong\langle\langle w, t\rangle\rangle$. Also, $\langle r, r v\rangle \cong\langle r, r w\rangle$ and thus

$$
\sigma \cong\langle 1, p, q, p q, r, r w\rangle \cong\langle 1, w, t, t w, r, r w\rangle .
$$

The case $\operatorname{dim} \sigma=7$. By the 6-dimensional case, we may assume after scaling that $\sigma \cong\langle 1, p, q, p q, r, p r, s\rangle \subset\langle\langle p, q, r\rangle\rangle$. We can write $s=u+v$ with $u \in$ $D_{F}(\langle q r, p q r\rangle)$ and $v \in D_{F}(\langle 1, p, q, p q, r, p r\rangle)$. The anisotropy implies $u \neq 0$ and we get $\sigma \cong\langle 1, p, q, p q, r, p r, u\rangle$. Now $q r u \in D_{F}(\langle 1, p\rangle)$ and thus, by Proposition 8.5 (or Proposition 8.1]), $u p\langle 1, p\rangle \cong u\langle 1, p\rangle \cong q r\langle 1, p\rangle$. It follows that

$$
u p \sigma \cong\langle p, q, r, p q, p r, q r, p q r\rangle .
$$


It should be noted that the description of quasi-Pfister neighbors in dimensions $5,6,7$ bears a striking resemblance to the well-known characterization of Pfister neighbors of the same dimensions in characteristic $\neq 2$ (see, e.g. [24, p. 10]). However, our methods to obtain the characterization are naturally very different from those usually employed in characteristic $\neq 2$.

Let us now give a necessary condition for the isotropy of an anisotropic totally singular form over the function field of another totally singular form, which then yields criteria for the isotropy of anisotropic quasi-Pfister forms over function fields of totally singular forms. Recall that anisotropic totally singular forms stay anisotropic over function fields of forms which are not totally singular, so there is no need to consider this case.

Proposition 8.13. Let $\varphi, \psi$ be nonzero totally singular forms with $\varphi$ anisotropic. Let $K=F(\psi)$. If $\varphi_{K}$ is isotropic, then $N_{F}(\psi) \subset N_{F}(\varphi)$.

Proof. Without loss of generality, we may assume that $\psi$ is anisotropic, $\operatorname{dim} \psi \geq 2$, and, after scaling, $\psi \cong\left\langle 1, b_{1}, \cdots, b_{n}\right\rangle$.

Let $\operatorname{ndeg}_{F}(\varphi)=2^{m}$ and let $a_{1}, \cdots, a_{m} \in F^{*}$ such that $N_{F}(\varphi)=F^{2}\left(a_{1}, \cdots, a_{m}\right)$. Let $\pi=\left\langle\left\langle a_{1}, \cdots, a_{m}\right\rangle\right\rangle$. Then $\pi$ is anisotropic by Proposition 8.5 and by Proposition 8.6, $\varphi$ is similar to a subform of $\pi$. Hence, $\varphi_{K}$ isotropic implies that $\pi_{K}$ is isotropic.

Let $i \in\{1, \cdots, n\}$ and put $L^{\prime}=F\left(\left\langle 1, b_{i}\right\rangle\right)=F(X)\left(\sqrt{b_{i} X^{2}}\right.$ ) (where $X$ denotes a variable), $L=F\left(\sqrt{b_{i}}\right)$. By a standard specialization argument ([28, Lemma 4.5]), the isotropy of $\pi_{K}$ implies that of $\pi_{L^{\prime}}$, and therefore also that of $\pi_{L}$. Hence, necessarily $\operatorname{ndeg}_{L}\left(\pi_{L}\right)<\operatorname{ndeg}_{F}(\pi)=2^{m}$. But $N_{F}(\pi)=N_{F}(\varphi)=F^{2}\left(a_{1}, \cdots, a_{m}\right)$, and $N_{L}\left(\pi_{L}\right)=F^{2}\left(a_{1}, \cdots, a_{m}, b_{i}\right)$. If $b_{i} \notin F^{2}\left(a_{1}, \cdots, a_{m}\right)$, then $\left[F^{2}\left(a_{1}, \cdots, a_{m}, b_{i}\right)\right.$ : $\left.F^{2}\right]=2^{m+1}$ and thus $\left[F^{2}\left(a_{1}, \cdots, a_{m}, b_{i}\right): F^{2}\left(b_{i}\right)\right]=\left[L^{2}\left(a_{1}, \cdots, a_{m}\right): L^{2}\right]=$ $\operatorname{ndeg}_{L}\left(\pi_{L}\right)=2^{m}$, a contradiction. Thus, $b_{i} \in F^{2}\left(a_{1}, \cdots, a_{m}\right)$ for every $i$, hence we obtain

$$
N_{F}(\psi)=F^{2}\left(b_{1}, \cdots, b_{n}\right) \subset N_{F}(\varphi)
$$

Corollary 8.14. Let $\pi$ be an anisotropic $m$-fold quasi-Pfister form, $m \geq 1$, and let $\sigma$ be a totally singular form. Let $K=F(\sigma)$. The following are equivalent:

(i) $\pi_{K}$ is isotropic.

(ii) $\operatorname{dim} \sigma_{\text {an }} \geq 2$ and for any $a \in D_{F}^{*}(\sigma)$ one has $a \sigma_{\text {an }} \subset \pi$.

(iii) $\operatorname{dim} \sigma_{\text {an }} \geq 2$ and $N_{F}(\sigma) \subset N_{F}(\pi)$.

Proof. Since $F(\sigma) / F\left(\sigma_{\text {an }}\right)$ is purely transcendental, we have that $\pi_{K}$ is isotropic iff $\pi_{F\left(\sigma_{\text {an }}\right)}$ is isotropic. Also, $N_{F}(\sigma)=N_{F}\left(\sigma_{\text {an }}\right)$, so we may assume, without loss of generality, that $\sigma$ is anisotropic.

By the previous proposition, (i) implies (iii). (iii) implies (ii) by Propositions 8.5 and 8.6, and trivially (ii) implies (i).

We conclude this section with some remarks on splitting towers and heights of forms (singular or not). One of the important properties of generic splitting towers in characteristic $\neq 2$ is the fact that if $\varphi$ is an anisotropic form over the field $E$, and if $K$ is any field extension of $E$, then there exists a field $E_{i}$ in the generic splitting tower $E=E_{0} \subset E_{1} \subset \cdots \subset E_{h}$ such that $\operatorname{dim}\left(\varphi_{K}\right)_{\text {an }}=\operatorname{dim}\left(\varphi_{E_{i}}\right)_{\text {an }}$.

This property no longer holds in general for standard splitting towers of totally singular forms as the following example shows. 
Example 8.15. Consider a totally singular anisotropic form $q=\left\langle\left\langle a_{1}, \cdots, a_{n}\right\rangle\right\rangle \perp$ $\langle b\rangle, n \geq 2$. Then $q$ is a quasi-Pfister neighbor of $\left\langle\left\langle a_{1}, \cdots, a_{n}, b\right\rangle\right\rangle$, and thus the standard splitting pattern will be $\left(2^{n}+1,2^{n}, 2^{n-1}, \cdots, 2,1\right)$ by the preceding theorem. Now for $K_{m}=F\left(\sqrt{a_{1}}, \cdots, \sqrt{a_{m}}\right), 1 \leq m \leq n-1$, it is not difficult to show that $\left(q_{K_{m}}\right)_{\text {an }} \cong\left\langle\left\langle a_{m+1}, \cdots, a_{n}\right\rangle\right\rangle \perp\langle b\rangle$, and it follows that $2^{n-m}+1=\operatorname{dim}\left(q_{K_{m}}\right)_{\text {an }}$ does not show up in the standard splitting pattern.

However, we do know by Proposition 4.6 that the dimension of the nonsingular part of the anisotropic part of a form over a field extension will appear as dimension of the nonsingular part of one of its higher kernel forms in the standard splitting tower. With our results on totally singular forms over field extensions, we are now able to prove Theorem 4.5 which shows that the maximal height of a tower of fields over which the anisotropic parts of a form are of strictly decreasing dimension is given by the standard height. We restate the theorem for the reader's convenience.

Theorem 8.16. Let $q$ be an anisotropic form of standard height $h(q)=h$. Let $F=$ $K_{0} \subset K_{1} \subset \cdots \subset K_{m}$ be any tower of fields such that $\operatorname{dim}\left(q_{K_{i-1}}\right)_{\text {an }}>\operatorname{dim}\left(q_{K_{i}}\right)_{\text {an }}$ for each $i \in\{1, \cdots, m\}$. Then $m \leq h$.

Proof. Let $q_{(i)}=\left(q_{K_{i}}\right)_{\text {an }}$ and denote by $\left(r_{i}, s_{i}\right)$ its type. By assumption, $\operatorname{dim} q_{(i)}<$ $\operatorname{dim} q_{(i-1)}$ and thus $r_{i}<r_{i-1}$ or $s_{i}<s_{i-1}$ for $1 \leq i \leq m$. Let $u=\operatorname{card}\{i \mid 1 \leq i \leq$ $\left.m, r_{i}<r_{i-1}\right\}$ and $v=\operatorname{card}\left\{i \mid 1 \leq i \leq m, s_{i}<s_{i-1}\right\}$. Then $u+v \geq m$. On the other hand, by Proposition 4.6 we have $u \leq h_{\text {nd }}(q)$.

Let $\sigma_{i}=\mathrm{ql}\left(q_{(i)}\right)$ and $\sigma=\sigma_{0}=\mathrm{ql}(q)$. Now $\sigma_{i}=\left(\sigma_{K_{i}}\right)_{\text {an }}$ and $\operatorname{dim} \sigma_{i}=s_{i}$. If $\operatorname{ndeg}_{K_{i}}\left(\sigma_{i}\right)=2^{m_{i}}$ and $s_{i}<s_{i-1}$, then by Proposition $8.7 m_{i}<m_{i-1}$ because the anisotropic form $\sigma_{i-1}$ becomes isotropic over $K_{i}$. Consequently, $v \leq m_{0}$. But $h(\sigma)=m_{0}$ by Theorem 8.11 (i), and therefore $v \leq h(\sigma)$. We have $h(q)=h_{\mathrm{nd}}(q)+$ $h(\sigma)$ (cf. [29, Th. 4.6]), hence

$$
h(q) \geq u+v \geq m
$$

\section{ACKNOWLEDGEMENT}

The authors thank Manfred Knebusch for many valuable comments which led to various improvements in the final version, and also the referee who pointed out to us Corollary 5.3 which replaces a weaker result in an earlier version of the present paper.

\section{REFERENCES}

1. H. Ahmad, The algebraic closure in function fields of quadratic forms in characteristic 2, Bull. Austral. Math. Soc. 55 (1997), 293-297. MR 98a:11042

2. A.A. Albert, Structure of Algebras, AMS Colloq. Publ. XXIV, Providence, RI, 1939. MR $1: 99 \mathrm{c}$

3. J.Kr. Arason, Cohomologische Invarianten quadratischer Formen, J. Algebra 36 (1975), 448491. MR 52:10592

4. J.Kr. Arason, R. Elman, Powers of the fundamental ideal in the Witt ring. J. Algebra 239 (2001), 150-160. MR 2002b:11054

5. J.Kr. Arason, A. Pfister, Beweis des Krullschen Durschnittsatzes für den Wittring. Invent. Math. 12 (1971), 173-176. MR 45:3320

6. R. Aravire, R. Baeza, A note on generic splitting of quadratic forms, Comm. Alg. 27 (1999), 3473-3477. MR 2000c:11062 
7. R. Aravire, R. Baeza, The behaviour of quadratic and differential forms under function field extensions in characteristic 2, J. Algebra 259 (2003), no. 2, 361-414.

8. R. Aravire, B. Jacob, P. Mammone, On the u-invariant for quadratic forms and the linkage of cyclic algebras, Math. Z. 214 (1993), 137-146. MR 94k:11045

9. C. Arf, Untersuchungen über quadratische Formen in Körpern de Characteristik 2 (Teil I), J. Reine Angew. Math. 183 (1941), 148-167. MR 4:237f

10. R. Baeza, Ein Teilformensatz für quadratische Formen in Characteristic 2, Math. Z. 135 (1974), 175-184. MR 49:2534

11. R. Baeza, Quadratic forms over semi-local rings, Lecture Notes in Mathematics, vol. 655, Springer-Verlag, 1976. MR 58:10972

12. R. Baeza, The norm theorem for quadratic forms over a field of characteristic 2, Comm. Alg. 18 (1990), 1337-1348. MR 91h:11024

13. J.-L. Colliot-Thélène, A.N. Skorobogatov, Groupe de Chow des zéro-cycles sur les fibres en quadriques, K-Theory 7 (1993), 477-500. MR 95c:14012

14. P.K. Draxl, Skew fields, London Math. Soc. Lecture Note Series, vol. 81, Cambridge University Press, Cambridge, 1983. MR 85a:16022

15. R. Fitzgerald, Function fields of quadratic forms, Math. Z. 178 (1981), 63-76. MR 83b:10021

16. D. Hoffmann, Isotropy of quadratic forms over the function field of a quadric, Math. Z. 220 (1995), 461-476. MR 96k:11041

17. D. Hoffmann, Splitting patterns and invariants of quadratic forms, Math. Nachr. 190 (1998), 149-168. MR 99g:11047

18. D. Hoffmann, A. Laghribi, Isotropy of quadratic forms over the function field of a quadric in characteristic 2, Preprint 2002.

19. J. Hurrelbrink, U. Rehmann, Splitting patterns of excellent quadratic forms, J. reine angew. Math. 444 (1993), 183-192. MR 94h:11030

20. J. Hurrelbrink, U. Rehmann, Splitting patterns of quadratic forms, Math. Nachr. 176 (1995), 111-127. MR 96k:11043

21. B. Kahn, Formes quadratiques de hauteur et de degré 2, Indag. Mathem., N.S. 7 (1996), 47-66. MR 99h:11042

22. M. Knebusch, Specialization of quadratic and symmetric bilinear forms, and a norm theorem, Acta Arithm. 24 (1973), 279-299. MR 50:2075

23. M. Knebusch, Generic splitting of quadratic forms I, Proc. London Math. Soc. 33 (1976), 65-93. MR 54:230

24. M. Knebusch, Generic splitting of quadratic forms II, Proc. London Math. Soc. 34 (1977), 1-31. MR 55:379

25. M. Knebusch, Spezialisierung von quadratischen und symmetrisch bilinearen Formen, book in preparation.

26. M. Knebusch, U. Rehmann, Generic splitting towers and generic splitting preparation of quadratic forms, Contemp. Math. 272 (2000), 173-199. MR 2001g:11052

27. M. Knebusch, W. Scharlau, Algebraic Theory of Quadratic Forms. Generic Methods and Pfister Forms, DMV Seminar 1, Birkhäuser, Boston, Mass., 1980 MR 82b:10023

28. A. Laghribi, Certaines formes quadratiques de dimension au plus 6 et corps de fonctions en caractéristique 2, Israel J. Math. 129 (2002), 317-362. MR 2003f:11047

29. A. Laghribi, On the generic splitting of quadratic forms in characteristic 2, Math. Z. 240 (2002), 711-730. MR 2003f:11048

30. A. Laghribi, On splitting of totally singular quadratic forms, preprint (2003).

31. A. Laghribi, P. Mammone, Isotropie d'une forme quadratique sur le corps des fonctions d'une quadrique projective en caractéristique 2, Bull. Belg. Math. Soc. 9 (2002), 167-176.

32. P. Mammone, J.-P. Tignol, A. Wadsworth, Fields of characteristic 2 with prescribed uinvariants, Math. Ann. 290 (1991), 109-128. MR 92g:11035

33. A.S. Merkurjev, Simple algebras and quadratic forms, Izv. Akad. Nauk. SSSR 55 (1991), 218-224. English translation: Math. USSR Izvestiya 38 (1992), 215-221. MR 93b:16025

34. D. Orlov, A. Vishik, V. Voevodsky, Motivic cohomology of Pfister quadrics and Milnor's conjecture on quadratic forms, preprint (1998).

35. A. Pfister, Quadratic Forms with Applications to Algebraic Geometry and Topology, London Math. Soc. Lecture Note Series, vol. 217, Cambridge University Press, Cambridge, 1995. MR 97c:11046 
36. C.-H. Sah, Symmetric bilinear forms and quadratic forms, J. Algebra 20 (1972), 144-160. MR 45:3448

37. W. Scharlau, Quadratic and Hermitian Forms, Grundlehren der math. Wissenschaften 270, Springer-Verlag, Berlin, Heidelberg, New York, 1985. MR 86k:11022

38. A.R. Wadsworth, Similarity of quadratic forms and isomorphisms of their function fields, Trans. Amer. Math. Soc. 208 (1975), 352-358. MR 51:12702]

Laboratoire de Mathématiques, UMR 6623 du CNRS, Université de Franche-Comté, 16 Route de Gray, F-25030 Besançon Cedex, France

E-mail address: detlev@math.univ-fcomte.fr

Current address: School of Mathematical Sciences, University of Nottingham, University Park, Nottingham NG7 2RD, Great Britain

E-mail address: detlev.hoffmann@nottingham.ac.uk

Fakultät für Mathematik, Universität Bielefeld, Postfach 100131, D-33501 BieleFELD, Germany

E-mail address: laghribi@mathematik.uni-bielefeld.de 